\title{
Resonances in the Stark Effect of Atomic Systems
}

\author{
S. Graffi and V. Grecchi` \\ Istituto Matematico, Università di Modena, I-41100 Modena, Italy
}

\begin{abstract}
Generalizing earlier results on the Hydrogen case it is proved, through a dilation analyticity technique different from the canonical one, that the action of a weak electric field shifts the isolated eigenvalues of any atomic system into resonances of the Stark effect, uniquely determined by the perturbation series through the Borel summation method.
\end{abstract}

\section{Introduction}

This paper represents a continuation of a preceding one [3] in which the existence of resonances in the Hydrogen Stark effect and the Borel summability of the divergent perturbation series were proved through the well known separability of the problem in squared parabolic coordinates. These results have been independently and almost simultaneously obtained by Herbst [5] for a two-body Stark operator with a dilation analytic potential of general type. Furthermore Herbst and Simon [6] have announced a generalization [7]; see also Hunziker [8] of Herbst's results to a $N$-body Schrödinger operator of the type

$$
H(F)=-\sum_{1}^{N} \Delta_{i}-\sum_{1}^{N} V_{i}\left(\mathbf{r}_{i}\right)+\sum_{i<k}^{N} V_{i k}\left(\mathbf{r}_{i}-\mathbf{r}_{k}\right)+\sum_{1}^{N} z_{i}
$$

which includes the operator describing the Stark effect on any atomic system obtained for $V\left(\mathbf{r}_{i}\right)=Z /\left|\mathbf{r}_{i}\right|, V_{i k}=1 /\left|\mathbf{r}_{i}-\mathbf{r}_{k}\right|$, if $Z$ is the atomic number, $F$ the electric field strength, and the electron charge is put equal to one.

The key argument of [5] is the discovery that the canonically dilated operator $-e^{-2 \phi} \Delta+F e^{\phi} z$ associated with $-\Delta+F z$ is, when defined on $D(-\Delta) \cap D(z)$, a holomorphic family of type $A$ with empty spectrum for $0<|\operatorname{Im} \phi|<\pi / 3$. This allows to extend the dilation analyticity technique $[1,2]$ to the Stark problem, which is not dilation analytic according to the usual notion of this concept (see e.g. Reed and Simon [10, Sect. XIII.10]).

* Also at Istituto di Fisica, Università di Modena 
Here on the contrary the basic argument is as in [3], although, of course, the problem is no longer separable. It consists indeed in proving the relevant analyticity statements by performing an anisotropic dilation on the squared parabolic coordinates (Sect. II). In the Hydrogen case the separability implies that the dilation analyticity in this sense is related to the well known analyticity of the anharmonic oscillator operator family [12]. In the present $N$-body case it has to be explicitly proved. This procedure allows (Sect. III) to associate with the operator $-\sum_{1}^{N} \Delta_{i}+\sum_{1}^{N} z_{i}$ a holomorphic family of $m$-sectorial operators with compact resolvents for suitable non real values of the dilation parameter. Then the standard hypothesis that the potentials are dilation analytic makes the introduction of the Weinberg-Van Winter equation unnecessary and yields the compactness of the resolvent of the dilated operator associated with $H(F)$ by the present procedure. The eigenvalues of this operator are identified with the resonances of $H(F)$. On the other hand (Sect. IV) the compactness and the sectoriality allow the application of the Weinberg-Van Winter equation to prove the convergence of the resonances to the atomic bound states (at least those lying below all thresholds) as $F \rightarrow 0$. Finally for any non degenerate bound state this stability result will be strengthened to the Borel summability [4] of its RayleighSchrödinger perturbation expansion to the nearby resonance.

\section{Parabolic Dilation Analyticity}

The formal Schrödinger operator describing the motion of a particle of unit charge and mass $1 / 2$ under the action of a uniform electric field of strength $F>0$ directed along the $z$ axis is:

$$
h_{0}(F)=-\Delta+F z \text {. }
$$

$h_{0}(F)$ is essentially self-adjoint in $L^{2}\left(R^{3}\right)$ if defined on $C_{0}^{\infty}\left(R^{3}\right)$. Its unitary image under the standard dilation map $(x, y, z)+\left(e^{\phi} x, e^{\phi} y, e^{\phi} z\right), \phi \in R$, is

$$
h_{0}(F, \phi)=-e^{-2 \phi} \Delta=e^{\phi} F z, \quad D\left(h_{0}(F, \phi)\right)=C_{0}^{\infty}\left(R^{3}\right) .
$$

The differential expression (2.2) can be formally continued to non real values of $\phi$. Its realization as an operator family in $L^{2}\left(R^{3}\right)$ has been given by Herbst [5]. Denoting (throughout this section) by $W_{m}$ the $m$-th Sobolev space on $R^{3}$, and by $L_{s}^{2}$ the weighted Hilbert space with weight $\left(1+|x|^{2}\right)^{s / 2}$, his results can be stated as follows:

Proposition 2.1. Let $0<|\operatorname{Im} \phi|<\pi / 3$, and $h_{0}(F, \phi)$ be defined as an operator in $L^{2}$ by the differential expression (2.2) with domain $W_{2} \cap D(z)$. Then:

(i) $h_{0}(F, \phi)$ is a holomorphic family of operators in $L^{2}$ (of type A: see e.g. $[9$, VII.1] for a definition).

(ii) $\sigma\left(h_{0}(F, \phi)\right)=\emptyset$.

(iii) If $\phi \rightarrow \phi_{0} \in R,\left[h_{0}(F, \phi)-E\right]^{-1} \rightarrow\left[\bar{h}_{0}\left(F, \phi_{0}\right)-E\right]^{-1}$, uniformly on compacts in $E$ for $\operatorname{Re} E<0, \operatorname{Im} E>0$.

Our first aim in this section is to prove an analogous result when the dilation is performed on a squared parabolic coordinate. To begin with, let us define the transformation and prove its unitarity. 
Lemma 2.2. Let $f(x, y, z)$ belong to $L^{2}$. Let $\theta \in R$, and $t(\theta): L^{2} \rightarrow L^{2}$ be defined as:

$$
\begin{gathered}
(t(\theta) f)(x, y, z)=e^{1 / 2 \theta}[r(\theta) / r]^{1 / 2} f\left(e^{1 / 2 \theta} x, e^{1 / 2 \theta} y, \alpha z+\beta r\right) \\
r=\left(x^{2}+y^{2}+z^{2}\right)^{1 / 2}, \quad r(\theta)=\alpha r+\beta z, \quad \alpha=\frac{1}{2}\left(1+e^{\theta}\right), \quad \beta=\frac{1}{2}\left(1-e^{\theta}\right) .
\end{gathered}
$$

Then $t(\theta)$ is a unitary map of $L^{2}$ onto itself.

Proof. We have of course $t(\theta)^{-1}=t(-\theta)$ with domain $L^{2}$. Then the unitarity follows just by remarking that the Jacobian of the transformation $(x, y, z) \rightarrow\left(e^{1 / 2 \theta} x\right.$, $\left.e^{1 / 2 \theta} y, \alpha z+\beta r\right)$ is $J(\theta)=e^{\theta}[r(\theta) / r]$. This proves Lemma 2.2.

Remarks. (i) Introduce the squared parabolic coordinates $(u, v, \phi): x=u v \cos \phi$, $y=u v \sin \phi, z=\frac{1}{2}\left(u^{2}-v^{2}\right) ; u^{2}=r+z, v^{2}=r-z, \phi=\operatorname{arctg}(y / x)$. Then $r=\frac{1}{2}\left(u^{2}+v^{2}\right)$, $r(\theta)=\frac{1}{2}\left(u^{2}+e^{\theta} v^{2}\right), t(\theta) u^{2} t(-\theta)=u^{2}, t(\theta) v^{2} t(-\theta)=e^{\theta} v^{2}$. We thus see that the transformation $t(\theta)$ amounts to a standard dilation performed only on the squared parabolic coordinate $v$.

(ii) Let $f(\theta)=[r(\theta) / r]^{1 / 2}$. Then the maximal multiplication operators by $f(\theta)$ and $f(\theta)^{-1}$ represent bounded holomorphic families [9, VII.1.1] in $L^{2}$ for all complex $\theta$ in the strip $|\operatorname{Im} \theta|<\pi$.

In order to obtain an explicit realization of the unitary image of the Laplace operator under $t(\theta)$, let us first prove a lemma which will be of fundamental importance in what follows.

Lemma 2.3. Let $h_{0}(\theta)$ be the operator family in $L^{2}$ defined as:

$$
\begin{aligned}
h_{0}(\theta)= & -\Delta-\left(e^{-\theta}-1\right)\left(\partial^{2} / \partial x^{2}+\partial^{2} / \partial y^{2}\right)-\frac{1}{2}\left(1-e^{-2 \theta}\right)(\alpha z+\beta r) r(\theta)^{-1} \partial^{2} / \partial z^{2} \\
& +\frac{1}{2}\left(1-e^{-2 \theta}\right) \partial^{2} / \partial z^{2}-\left(1-e^{-\theta}\right) r(\theta)^{-1} \partial / \partial z, \\
& -e^{-\theta} \beta\left(r r^{2}(\theta)\right)^{-1}(\alpha z+\beta r-2 r r(\theta) \partial / \partial z)\left(x \frac{\partial}{\partial x}+y \frac{\partial}{\partial y}\right) \quad D\left(h_{0}(\theta)\right)=W_{2} .
\end{aligned}
$$

Then for any $\varepsilon, 0<\varepsilon<\pi / 2$, there is $\delta>0$ such that $h_{0}(\theta)$ represents a holomorphic family (of type $A$ ) of $m$-sectorial operators for all complex $\theta$ in $|\theta|<\delta$, with opening angle not exceeding $2 \varepsilon$. The quadratic form domain of $h_{0}(\theta)$ for any such $\theta$ is $W_{1}$.

Proof. First remark that there is $\delta>0$ such that $r(\theta)^{-1}$ is holomorphic with $\left|r(\theta)^{-1}\right|<2 r^{-1}$ for $|\theta|<\delta$. Hence the operator $\left(1-e^{-\theta}\right) r(\theta)^{-1} \partial / \partial z$ is continuous from $W_{2}$ to $L^{2}$ because $\partial / \partial z$ is continuous from $W_{2}$ to $W_{1}$ and the multiplication by $r^{-1}$ is continuous from $W_{1}$ to $L^{2}$. Moreover we can always take $\delta$ such that $\left|(\alpha z+\beta r) r(\theta)^{-1}\right|<2$ for $|\theta|<\delta$, and hence $(\alpha z+\beta r) r(\theta)^{-1} \partial^{2} / \partial z^{2}$ is continuous from $W_{2}$ to $L^{2}$. Similar arguments apply to the other terms in $C(\theta)=h_{0}(\theta)+\Delta$. Therefore by taking $\delta$ suitably small we see that $C(\theta)$ defined on $W_{2}$ is relatively bounded, with relative bound smaller than 1 , with respect to $-\Delta$ both in the operator sense as well as in the quadratic forms one. Hence a direct application of standard perturbation arguments proves the lemma.

Theorem 2.4. Let $\theta \in R,|\theta|<\delta$, and $-\Delta(\theta)=-t(\theta) \Delta t(\theta)^{-1}$ be the unitary image of the Laplace operator under the map $t(\theta): L^{2} \leftrightarrow L^{2}$. Then, as an operator identity:

$$
-\Delta(\theta) \equiv f(\theta) h_{0}(\theta) f(\theta)^{-1},
$$

where $f(\theta)$ is as in Remark (ii) above. 
Proof. Let us first check that the transformation $f(\theta)^{-1} t(\theta) \psi,|\theta|<\delta$, leaves $W_{2}$ invariant. An easy computation shows that if $\psi \in W_{2},-\Delta\left(f(\theta)^{-1} t(\theta) \psi\right)$ $=f(\theta)^{-1} t(\theta) h_{0}(-\theta) \psi, h_{0}(-\theta)$ being given by the differential expression (2.4). Now $h_{0}(-\theta)$ has domain $W_{2}, t(\theta)$ is unitary and $f(\theta)^{-1}$ is bounded, so that $f(\theta)^{-1} t(\theta) \psi \in W_{2}$. The converse statement $\psi \in W_{2}$ if $f(\theta)^{-1} t(\theta) \psi$ is in $W_{2}$ is obtained by replacing $\theta$ by $-\theta$ in the above argument. Hence $D(\Delta(\theta)) \equiv t(\theta) W_{2}=f(\theta) W_{2}$. By definition, $D\left(f(\theta) h_{0}(\theta) f(\theta)^{-1}\right)=\left\{u \in L^{2} \mid f(\theta)^{-1} u \in W_{2}\right\}$ because $f(\theta)$ is bounded. Hence $D\left(f(\theta) h_{0}(\theta) f(\theta)^{-1}\right)=f(\theta) W_{2}=D(\Delta(\theta))$. Since one easily verifies that $-f(\theta) h_{0}(\theta) f(\theta)^{-1} \psi=\Delta(\theta) \psi$ when $\psi \in D(\Delta(\theta))$ the theorem is proved.

It is now possible to characterize the holomorphic operator family associated with $-\Delta$ by the analytic continuation of $t(\theta)$ to $\theta$ complex.

Corollary 2.5. Let $\theta$ be complex. Then there is $\delta>0$ such that the operator family $-\Delta(\theta)$ defined for $\theta \in R$ can be continued to a holomorphic family of operators in $L^{2}$ for all complex $\theta$ in $|\theta|<\delta$, and $\sigma(\Delta(\theta))=\sigma\left(h_{0}(\theta)\right)$.

Proof. Since $-\Delta(\theta)=f(\theta) h_{0}(\theta) f(\theta)^{-1}$ when $\theta \in R$, it is enough to show that the latter can be continued to a holomorphic family with non empty resolvent set for $|\theta|<\delta$. Then the assertion is a consequence of the unique continuation property of the holomorphic families [9, VII.1.2]. Now by Lemma 2.3 and Remark (ii) after Lemma $2.2 f(\theta) h_{0}(\theta) f(\theta)^{-1}$ defined on $\left\{u \in L^{2} \mid f(\theta)^{-1} u \in W_{2}\right\}$ is a closed operator for all complex $\theta$ in $|\theta|<\delta$ and $\left[f(\theta) h_{0}(\theta) f(\theta)^{-1}-E\right]^{-1} \equiv f(\theta)\left[h_{0}(\theta)-E\right]^{-1} f(\theta)^{-1}$ is a bounded holomorphic family for $|\theta|<\delta$ when $E \notin W\left(h_{0}(\theta)\right)$, the numerical range of $h_{0}(\theta)$. Hence $f(\theta) h_{0}(\theta) f(\theta)^{-1}$ is a holomorphic family, and the assertion on $\sigma(\Delta(\theta))$ is an immediate consequence of the above identity. This proves the corollary.

The second step consists in analyzing the operator associated with $-e^{-2 \phi} \Delta$ $+F e^{\phi} z$ along the lines of the above treatment of the Laplace operator.

A first obvious remark is that $t(\theta) z t(\theta)^{-1} \equiv \alpha z+\beta r$ as a multiplication operator in $L^{2}$, so that this operator can be extended to $\theta$ complex. For $0<\operatorname{Im} \theta<\pi$ one easily shows that the domain of the maximal multiplication operator by $\alpha z+\beta r$ is $L_{1}^{2}$. Then we have:

Theorem 2.6. Let $h_{0}(\gamma, \theta)$ be the operator family in $L^{2}$ defined as

$$
h_{0}(\gamma, \theta)=h_{0}(\theta)+\gamma(\alpha z+\beta r), \quad D\left(h_{0}(\gamma, \theta)\right)=W_{2} \cap L_{1}^{2} .
$$

(i) For any fixed $\gamma$ in $\{|\gamma|>0 \mid 0<\arg (\gamma)<\pi\}$ there are $\tilde{\theta}>0$ and an open set $M$ contained in $|\theta|<\delta, \delta$ as in Lemma 2.3, whose intersection with the imaginary $\theta$ axis contains the open interval $(0, \tilde{\theta})$, such that $h_{0}(\gamma, \theta)$ represents in $M$ a holomorphic family of type $A$ of $m$-sectorial operators with compact resolvents.

(ii) When $\arg (\gamma)=\pi / 2, \theta \in M, \operatorname{Re} \theta=0,\left[h_{0}(\gamma, \theta)-E\right]^{-1} \underset{s}{\rightarrow}[-\Delta+\gamma z-E]^{-1}$, uniformly on compacts in $E$ for $\operatorname{Re} E<0, \operatorname{Im} E>0$, as $\operatorname{Im} \theta \rightarrow 0$, and to $\left[h_{0}(\theta)-E\right]^{-1}$ as $|\gamma| \rightarrow 0,0<\arg (\gamma)<\pi, \theta \in M$, with the same uniformity in $E$.

Remark. From now on, when referring to $h_{0}(\gamma, \theta)$ we shall always assume that the conditions $\theta \in M, 0<\arg (\gamma)<\pi,|\gamma|>0$ are fulfilled.

The proof of the above theorem is to be obtained through the following lemmas, which are moreover of some relevance of their own in what follows. 
Lemma 2.7. Let $\varepsilon, \delta$ be as in Lemma 2.3. Then the numerical range $W\left(h_{0}(\gamma, \theta)\right)$ of $h_{0}(\gamma, \theta)$ is contained in a sector of opening angle strictly less than $\pi$. If $\arg (\gamma)=\pi / 2$, $W\left(h_{0}(\gamma, \theta)\right)=\{E \mid-\pi / 2+\operatorname{Im} \theta \leqq \arg (E) \leqq \pi / 2\}$.

Proof. The numerical range of $\gamma(\alpha z+\beta r)$ is contained in $\{E \mid-\pi=\operatorname{Im} \theta=\arg (\gamma)$ $\leqq \arg (E) \leqq \arg (\gamma)\}$ for all $\theta \in M$, because in squared parabolic coordinates $\alpha z+\beta r=u^{2}-e^{\theta} v^{2}$. Since for $|\theta|<\delta W\left(h_{0}(\theta)\right)$ is contained in a sector of opening angle $2 \varepsilon$ about the real axis the result is proved.

Lemma 2.8. Let $\theta$ and $\gamma$ be complex, $\theta \in M,|\gamma|>0,0<\arg (\gamma)<\pi$. Then if $\psi \in W_{2} \cap L_{1}^{2}$ there are $a>0, b>0, c>0$, independent of $(\gamma, \theta)$ on compacts of the stated regions, such that the following quadratic estimate holds:

$$
\|\{-\Delta+\gamma(\alpha z+\beta r)\} \psi\|^{2}+b\|\psi\|^{2} \geqq a\left\{\|-\Delta \psi\|^{2}+c\|\gamma r \psi\|^{2}\right\} .
$$

Proof. Proceeding as in [5], let us write, as quadratic forms on $W_{2} \cap L_{1}^{2} \otimes W_{2} \cap L_{1}^{2}$ (here $p^{2}=-\Delta$, and $p, p_{r}, p_{z}$ have their usual meaning): $\left(p^{2}+\bar{\gamma}(\bar{\alpha} z+\bar{\beta} r)\right)\left(p^{2}+\gamma(\alpha z+\beta r)\right)$ $=p^{4}+|\alpha \gamma|^{2} z^{2}+|\beta \gamma|^{2} r^{2}+2|\gamma|^{2} \operatorname{Re}(\bar{\alpha} \beta) r z+\operatorname{Re}(\alpha \gamma)\left(z p^{2}+p^{2} z\right)+\operatorname{Re}(\beta \gamma)\left(r p^{2}+p^{2} r\right)$ $+2 \operatorname{Im}(\alpha \gamma) p_{z}+2 \operatorname{Im}(\beta \gamma) p_{r}=$ (remember that $r p^{2}+p^{2} r=\left[p_{r},\left[p_{r}, r\right]\right]+2 \mathbf{p} \cdot(r \mathbf{p})$ $\left.=2 \mathbf{p} \cdot(r \mathbf{p}), z p^{2}+p^{2} z=2 \mathbf{p} \cdot(z \mathbf{p})\right)=p^{4}+|\alpha \gamma|^{2} z^{2}+|\beta \gamma|^{2} r^{2}+2|\gamma|^{2} \operatorname{Re}(\bar{\alpha} \beta) r z+2 \operatorname{Re}(\alpha \gamma) \mathbf{p}$ $\cdot(z \mathbf{p})+2 \operatorname{Re}(\beta \gamma) \mathbf{p} \cdot(r \mathbf{p})+2 \operatorname{Im}(\alpha \gamma) p_{z}+2 \operatorname{Im}(\beta \gamma) p_{r}=p^{4}+|\alpha \gamma|^{2} z^{2}+|\beta \gamma|^{2} r^{2}+2|\gamma|^{2} \operatorname{Re}(\bar{\alpha} \beta) r z$ $+2\left(\operatorname{Re}(\alpha \gamma) \mp \operatorname{Re}(\beta \gamma) \mathbf{p} \cdot(z \mathbf{p})+2 \operatorname{Re}(\beta \gamma) \mathbf{p} \cdot(r \pm z) \mathbf{p}+2 \operatorname{Im}(\alpha \gamma) p_{z}+2 \operatorname{Im}(\beta \gamma) p_{r} \geqq p^{4}\right.$ $+|\alpha \gamma|^{2} z^{2}+|\beta \gamma|^{2} r^{2}+2|\gamma|^{2} \operatorname{Re}(\bar{\alpha} \beta) r z+2(\operatorname{Re}(\alpha \gamma) \mp \operatorname{Re}(\beta \gamma)) \mathbf{p} \cdot(z \mathbf{p})+2 \operatorname{Im}(\alpha \gamma) p_{z}+2 \operatorname{Im}(\beta \gamma) p_{r}$. Now for any $\varepsilon, 0<\varepsilon<1$ and any $k \in R$ we have $2 k \mathbf{p} \cdot(z \mathbf{p}) \geqq-(1-\varepsilon)\left(p^{4}+k^{2}(1-\varepsilon)^{-2} z^{2}\right)$, So that: $\left(p^{2}+\bar{\gamma}(\bar{\alpha} z+\bar{\beta} r)\right)\left(p^{2}+\gamma(\alpha z+\beta r)\right) \geqq \varepsilon p^{4}-(1-\varepsilon)^{-1} \quad(\operatorname{Re}(\alpha \gamma)-\operatorname{Re}(\beta \gamma))^{2} z^{2}$ $+|\alpha \gamma|^{2} z^{2}+|\beta \gamma|^{2} r^{2}+2|\gamma|^{2} \operatorname{Re}(\bar{\alpha} \beta) r z+2 \operatorname{Im}(\alpha \gamma) p_{z}+2 \operatorname{Im}(\beta \gamma) p_{r} \geqq \eta p^{4}-(1-\varepsilon)^{-1}(\operatorname{Re}(\alpha \gamma)$ $-\operatorname{Re}(\beta \gamma))^{2} z^{2}+|\alpha \gamma|^{2}+|\beta \gamma|^{2} r^{2}+2|\gamma|^{2} \operatorname{Re}(\bar{\alpha} \beta) r z-b$, for some positive constants $\eta<\varepsilon$ and $b$ which can be chosen independent of $(\gamma, \theta)$ as required; $\eta$ can be taken arbitrarily close to $\varepsilon$ by taking $b$ large enough. Let now $\chi=\arg (\gamma), \theta=\theta_{1}+i \theta_{2}$. Then $\operatorname{Re}(\alpha \gamma)-\operatorname{Re}(\beta \gamma)=|\gamma| e^{\theta_{1}} \cos \left(\chi+\theta_{2}\right), 4|\alpha \gamma|^{2}=|\gamma|^{2}\left\{\left(1-e^{\theta_{1}} \cos \theta_{2}\right)^{2}=e^{2 \theta_{1}} \sin ^{2} \theta_{2}\right\}$, $4|\beta \gamma|^{2}=|\gamma|^{2} \quad\left\{\left(1-e^{\theta_{1}} \cos \theta_{2}\right)^{2}=e^{2 \theta_{1}} \sin ^{2} \theta_{2}\right\}, \quad 4|\gamma|^{2} \operatorname{Re}(\bar{\alpha} \beta)=|\gamma|^{2} \quad\left(1-e^{2 \theta_{1}}\right)$. Hence $4\left(p^{2}+\bar{\gamma}(\bar{\alpha} z+\bar{\beta} r)\right)\left(p^{2}+\gamma(\alpha z+\beta r)\right) \geqq 4 \eta p^{4}+2|\gamma|^{2}\left(1-e^{2 \theta_{1}}\right) r z+|\gamma|^{2}\left(1=e^{2 \theta_{1}}+2 e^{\theta_{1}} \cos \theta_{2}\right.$ $\left.-4(1-\varepsilon)^{-1} e^{2 \theta_{1}} \cos ^{2}\left(\chi+\theta_{2}\right)\right) z^{2}+|\gamma|^{2}\left(1=e^{2 \theta_{1}}-2 e^{\theta_{1}} \cos \theta_{2}\right) r^{2}$ with $1=e^{2 \theta_{1}}+2 e^{\theta_{1}} \cos \theta_{2}$ $\geqq\left(1+e^{\theta_{1}} \cos \theta_{2}\right)^{2} \geqq \min \left(4 e^{2 \theta_{1}} \cos ^{2} \theta_{2}, 4 \cos ^{2} \theta_{2}\right)$. Let now $m<1$, and choose $\varepsilon$ such that $\cos ^{2} \chi<m(1-\varepsilon)$. Hence there are $m_{1}<1$ and $\tilde{\theta}_{2}$ such that $(1-\varepsilon)^{-1} \cos ^{2}\left(\chi+\theta_{2}\right)$ $<m_{1} \cos ^{2} \theta_{2}$ for all $\theta_{2}$ in $0<\theta_{2}<\tilde{\theta}_{2}$. Since in the same range of $\Theta_{2}$ one has $\left(1-\cos \theta_{2}\right)>0$, we can always find $\tilde{\theta}_{1}\left(\tilde{\theta}_{2}\right)$ such that, for $\left|\theta_{1}\right|<\tilde{\theta}_{1}, 2\left|1-e^{2 \theta_{1}}\right|<\left(1+e^{2 \theta_{1}}\right.$ $\left.-2 e^{\theta_{1}} \quad \cos \theta_{2}\right)$. Since $|z| \leqq r$ this yields $\left(p^{2}+\bar{\gamma}(\bar{\alpha} z+\bar{\beta} r)\right) \quad\left(p^{2}+\gamma(\alpha z+\beta r)\right) \geqq \eta p^{4}$ $+|\gamma|^{2} q\left(1-\cos \theta_{2}\right) r^{2}$, for some $q>0$, which proves the result.

Lemma 2.9. The quadratic estimate (2.7) remains valid if we replace $-\Delta$ by $h_{0}(\theta)$, i.e. with possibly different constants $a>0, h>0, c>0$ but with the same uniformities for any $\psi \in W_{2} \cap L_{1}^{2}$ one has:

$$
\left\|\left\{h_{0}(\theta)+\gamma(\alpha z+\beta r)\right\} \psi\right\|^{2}+b\|\psi\|^{2} \geqq a\left\{\left\|h_{0}(\theta) \psi\right\|^{2}+c\|\gamma r \psi\|^{2}\right\} .
$$

Proof. As in Lemma 2.3, write $h_{0}(\theta)=-\Delta+C(\theta)$, where $C(\theta)$ is relatively bounded with respect to $-\Delta$ with relative bound arbitrarily small for $|\theta|$ suitably small. Then, for $\psi \in W_{2} \cap L_{1}^{2}:\left\|\left\{h_{0}(\theta)+\gamma(\alpha z+\beta r)\right\} \psi\right\| \geqq\|\{-\Delta+\gamma(\alpha z+\beta r)\} \psi\|-\|C(\theta) \psi\|$ $\geqq\|\{-\Delta+\gamma(\alpha z+\beta r)\} \psi\|-\varepsilon_{1}\|-\Delta \psi\|-b_{1}\|\psi\|$. The quadratic estimate (2.7) implies 
that there are $a_{2}, b_{2}, c_{2}$ such that $\|\{-\Delta+\gamma(\alpha z+\beta r)\} \psi\| \geqq a_{2}\|-\Delta \psi\|+c_{2}\|\gamma r \psi\|$ $-b_{2}\|\psi\|$. Since we can always find values of $\theta$ such that $\varepsilon_{1}<a_{2}$, we get $\|\left\{h_{0}(\theta)\right.$ $+\gamma(\alpha z+\beta r)\} \psi\left\|+\left(b_{1}+b_{2}\right)\right\| \psi\left\|\geqq\left(a_{2}-\varepsilon_{1}\right)\right\|-\Delta \psi\left\|+c_{2}\right\| \gamma r \psi \|$, whence the result because, again, there are $\varepsilon_{2}, b_{3}>0$ such that $\left(1+\varepsilon_{2}\right)\|-\Delta \psi\| \geqq\left\|h_{0}(\theta) \psi\right\|+b_{3}\|\psi\|$.

Proof of Theorem 2.6. We already know that $Q\left(h_{0}(\theta)\right)=W_{1}$, and clearly one has $Q(\gamma(\alpha z+\beta r))=L_{1 / 2}^{2}$. If we choose $\varepsilon<\arg (\gamma)<\pi-\varepsilon, 2 \varepsilon$ the opening angle of Lemma 2.3, both forms $e^{i(\pi / 2-\arg (\gamma))} h_{0}(\theta)$ and $e^{i(\pi / 2-\arg (\gamma))} \gamma(\alpha z+\beta r)$ are strictly sectorial with numberical ranges contained in the right half-plane Re $H \geqq 0$. Hence we can define the form sum $\tilde{h}_{0}(\gamma, \theta)$ of $h_{0}(\theta)$ and $\gamma(\alpha z+\beta r)$ as the operator associated with the sum of the two above forms. $\tilde{h}_{0}(\gamma, \theta)$ is $m$-sectorial and $Q\left(\tilde{h}_{0}(\gamma, \theta)\right)=W_{1} \cap L_{1 / 2}^{2}$. Furthermore, $\tilde{h}_{0}(\gamma, \theta) \supset h_{0}(\gamma, \theta)$. Now the quadratic estimate (2-8) implies that $h_{0}(\gamma, \theta)$ is closed on $W_{2} \cap L_{1}^{2}$, which is dense in $W_{1} \cap L_{1 / 2}^{1}$ in the norm of $W_{1} \cap L_{1 / 2}^{2}$ and hence is a form core of $\tilde{h}_{0}(\gamma, \theta)$. Therefore (see e.g. [9, VI.2.1]) $\tilde{h}_{0}(\gamma, \theta)=h_{0}(\gamma, \theta)$. Now by the closed graph theorem the operator $\left[h_{0}(\gamma, \theta)-E\right]^{-1}(-\Delta+r+1)$ is bounded for $E \notin W\left(h_{0}(\gamma, \theta)\right)$. Hence $\left[h_{0}(\gamma, \theta)-E\right]^{-1} \equiv\left[h_{0}(\gamma, \theta)-E\right]^{-1}(-\Delta+r+1)$ $(-\Delta+r+1)^{-1}$ is compact by the compactness of $(-\Delta+r+1)^{-1}$. This proves assertion (i). To see (ii), remark that the union over $\operatorname{Im}\left(\theta \geqq 0\right.$ of $W\left(h_{0}(\gamma, \theta)\right)$ is contained in the half-plane $\operatorname{Re} E>0$ when $\arg (\gamma)=\pi / 2,|\theta|<\delta$. Hence $\left[h_{0}(\gamma, \theta)\right.$ $-E]^{-1}$ is bounded uniformly over $\operatorname{Im} \theta>0, \operatorname{Re} \theta=0$ when $\arg (\gamma)=\pi / 2$ and thus its strong convergence to $(-\Delta+\gamma z-E)^{-1}$ with the stated uniformality as $\operatorname{Im} \theta \rightarrow 0$ follows by a direct application of a known result [9, Theorem ViII.1.5] because $h_{0}(\gamma, \theta) u \rightarrow(-\Delta+\gamma z) u$ when $\operatorname{Re} \theta=0, \arg (\gamma)=\pi / 2, \operatorname{Im} \theta \rightarrow 0$, for any $\psi \in W_{2} \cap L_{1}^{2}$ which is a core of $-\Delta+\gamma z$. Analogously, since the union over $|\gamma| \geqq 0$ of $W\left(e^{i(\pi / 2-\arg (\gamma))} h_{0}(\gamma, \theta)\right)$ is contained in $\operatorname{Re} E \geqq 0$ and $h_{0}(\gamma, \theta) u \rightarrow h_{0}(\theta) u$ as $|\gamma| \rightarrow 0$ when $u \in C_{0}^{\infty}$ which is a core of $h_{0}(\theta),\left[h_{0}(\gamma, \theta)-E\right]^{-1} \rightarrow\left[h_{0}(\theta)-E\right]^{-1}$ as $|\gamma| \rightarrow 0$ with the stated uniformity. This proves Theorem 2.6.

From now on let us call a dilation analytic vector in the standard sense a canonical dilation analytic vector. If $\psi \in L^{2}$ is such that any scalar product $\langle t(\theta) \psi, \phi\rangle \equiv\langle\psi(\theta), \phi\rangle, \phi \in L^{2}$, defines an analytic function of $\theta$ in some strip $|\operatorname{Im} \theta|<\delta, \delta>0$, it will be called a parabolic dilation analytic vector. It is immediate to check that, given $\delta>0$, there always is a dense set of parabolic dilation analytic vectors.

Corollary 2.10. Let $\delta>0$.

(i) If $\psi_{1}, \psi_{2}$ are parabolic dilation analytic vectors for $|\operatorname{Im} \theta|<\delta$, then, for $\arg (\gamma)=\pi / 2$ :

$$
\left\langle\psi_{1},(-\Delta+\gamma z-E)^{-1} \psi_{2}\right\rangle=\left\langle\psi_{1}(\bar{\theta}), f(\theta)\left[h_{0}(\gamma, \theta)-E\right]^{-1} f(\theta)^{-1} \psi_{2}(\theta)\right\rangle .
$$

(ii) $\sigma\left(h_{0}(\gamma, \theta)\right)=\emptyset$ for $\arg (\gamma)=\pi / 2$.

(iii) If $\psi_{1}, \psi_{2}$ are jointly canonical and parabolic dilation analytic i.e. the scalar products $\left\langle t(\theta) u(\phi) \psi_{1}, \psi\right\rangle=\left\langle u(\phi) t(\theta) \psi_{1}, \psi\right\rangle, u(\phi)$ being the canonical dilation, are analytic functions of $(\theta, \phi)$ in $\{(|\operatorname{Im} \theta|<\delta) \times(|\operatorname{Im} \phi|<\eta)\}, \eta>0$, we have, for $0<\arg \left(F e^{3 \phi}\right)<\pi$ :

$$
\left\langle\psi_{1},(-\Delta+F z-E)^{-1} \psi_{2}\right\rangle=\left\langle\psi_{1}(\bar{\theta}, \bar{\phi}),\left[e^{-2 \phi} h_{0}\left(F e^{3 \phi}, \theta\right)-E\right]^{-1} \psi_{2}(\theta, \phi)\right\rangle .
$$

Proof. (i) The operator family $f(\theta)\left[h_{0}(\gamma, \theta)-E\right]^{-1} f(\theta)^{-1}$ is of course holomorphic in the same holomorphy region of $\left[h_{0}(\gamma, \theta)-E\right]^{-1}$. On the other hand, the scalar 
products in the r.h.s. of (2.9) are independent of $\theta$ for $\theta \in M$ because $\left[f(\theta) h_{0}(\theta) f(\theta)^{-1}\right.$ $\left.+f(\theta) \gamma(\alpha z+\beta r) f(\theta)^{-1}-E\right]^{-1} \equiv f(\theta)\left[h_{0}(\gamma, \theta)-E\right]^{-1} f(\theta)^{-1}$ (which can be proved exactly as in Theorem 2.4), the unitary equivalence between $f\left(\theta+\theta_{1}\right) h_{0}$ $\cdot\left(\gamma, \theta+\theta_{1}\right) f\left(\theta+\theta_{1}\right)^{-1}$ and $f(\theta) h_{0}(\theta) f(\theta)^{-1}$ for $\theta_{1} \in R$ and the usual analyticity argument. Hence (2.9) is simply a consequence of the strong convergence of Theorem 2.6(ii). Assertion (ii) is a consequence of $\sigma(-\Delta+\gamma z)=\emptyset[5$, II.1] and of the compactness of $\left[h_{0}(\gamma, \theta)-E\right]^{-1}$ because (2.9) holds for any $\psi_{1}, \psi_{2}$ belonging to a dense set. Finally, (iii) is a consequence of (2.9) and of Herbst's result if we set $\gamma=F e^{3 \phi}$. This proves Corollary 2.10 .

\section{Resonances in the $N$-Body Case}

The formal Schrödinger operator in $L^{2}\left(R^{3 N}\right)$ describing the motion of $N$ particles of mass $1 / 2$ and unit charge under the action of a uniform electric field of strength $F>0$ directed along the $z$ axis is

$$
H_{0}(F)=-\sum_{1}^{N} \Delta_{i}+F \sum_{1}^{N} z_{i}
$$

where $r_{i}=\left(x_{i}, y_{i}, z_{i}\right)$ denotes the position of the $i$-th particle, and $\Delta_{i}$ the Laplace operator with respect to $\left(x_{i}, y_{i}, z_{i}\right)$. If in addition the particles interact via twobody potentials $V_{i j}\left(\mathbf{r}_{i}-\mathbf{r}_{j}\right)$, and each particle interacts with a fixed centre via a potential $V_{i}\left(\mathbf{r}_{i}\right)$, we are led to the formal Schrödinger operator for the $N$-body Stark effect:

$$
H(F)=-\sum_{1}^{N} \Delta_{i}+\sum_{1}^{N} V\left(\mathbf{r}_{i}\right)+\sum_{i<j}^{N} V_{i j}\left(\mathbf{r}_{i}-\mathbf{r}_{j}\right)+F \sum_{1}^{N} z_{i}
$$

We will now describe the realization of the various holomorphic families of operators associated with $H_{0}(F)$ and (under suitable assumptions on the potentials to be stated below) with $H(F)$ by the analytic continuation of the unitary dilation, formulated both in the canonical way as well as in terms of the squared parabolic coordinates. Consider in $L^{2}\left(R^{3 N}\right)$ the essentially self-adjoint operator $H_{0}(F)$ defined by (3.1) on $C_{0}^{\infty}\left(R^{3 N}\right)$. Its unitary image under the dilation map $\left(x_{i}, y_{i}, z_{i}\right)$ $\rightarrow\left(e^{\phi} x_{i}, e^{\phi} y_{i}, e^{\phi} z_{i}\right), \phi \in R, i=1, \ldots, N$, is given by $T_{0}(F, \phi)=-e^{-2 \phi} \sum_{1}^{N} \Delta_{i}+e^{\phi} F \sum_{1}^{N} z_{i}$ with domain $C_{0}^{\infty}\left(R^{3 N}\right)$. With $\phi$ is complex, we have a straightforward generalization of Herbst's characterization of $-e^{-2 \phi} \Delta+e^{\phi} F z$ in the two-body case, which for convenience is stated as a lemma, with proof of course omitted.

Lemma 3.1. Let $0<\arg (F)+3 \operatorname{Im} \phi<\pi$, and let $T_{0}(F, \phi)$ be defined as an operator in $L^{2}\left(R^{3 N}\right)$ by

$$
T_{0}(F, \phi)=-e^{-2 \phi} \sum_{1}^{N} \Delta_{i}+e^{\phi} F \sum_{2}^{N} z_{i}, \quad D\left(T_{0}(F, \phi)\right)=W_{2}\left(R^{3 N}\right) \cap D\left(\sum_{1}^{N} z_{i}\right) .
$$

Then:

(i) $T_{0}(F, \phi)$ is a holomorphic family of type $A$ of operators in $L^{2}\left(R^{3 N}\right)$ (in each single variable, the other one being held fixed) with empty spectrum. 
(ii) For $\arg (F)=0,\left[T_{0}(F, \phi)-E\right]^{-1} \rightarrow\left[\bar{T}_{0}\left(F, \phi_{0}\right)-E\right]^{-1}$ as $\phi \rightarrow \phi_{0} \in R$, uniformly on compacts in $E$ for $\operatorname{Re} E<0, \operatorname{Im} E>0$.

In order to introduce the parabolic dilation analyticity in the present $N$-body case, let us first generalize the dilation operation of Lemma 2.2.

Lemma 3.2. Let $\theta \in R$, and $U_{0}(\theta)$ be the operator in $L^{2}\left(R^{3 N}\right)$ defined as $U_{0}(\theta)$ $=\bigotimes^{N} t_{i}(\theta)$, where $t_{i}(\theta)$ is the unitary operator in $L^{2}\left(R^{3}\right)$ of Lemma 2.2. Then $U(\theta)$ $=\bar{U}_{0}^{1}(\theta)$ is a unitary map of $L^{2}\left(R^{3 N}\right)$ onto itself.

Proof. Let $f_{i} \in L^{2}\left(R^{3}\right), i=1, \ldots, N$, and $f=\bigotimes_{1}^{N} f_{i} \in L^{2}\left(R^{3 N}\right)$. Then $\left\|U_{0}(\theta) f\right\|$ $=\left\|t(\theta) f_{1} \otimes t(\theta) f_{2} \otimes \ldots \otimes t(\theta) f_{N}\right\|=\|f\|=\left\|t(-\theta) f_{1} \otimes t(-\theta) f_{2} \otimes \ldots \otimes t(-\theta) f_{N}\right\|$ $=\left\|U_{0}(-\theta) f\right\|$. Since $U_{0}(-\theta)=U_{0}(\theta)^{-1}$ and $L^{2}\left(R^{3 N}\right) \cong \bigotimes_{1}^{N} L^{2}\left(R^{3}\right)$, the lemma is proved.

The generalization to the $N$-body case of the relevant properties of the parabolic dilation analyticity is simple. Introducing for simplicity the notations $\sum_{1}^{N} \Delta_{i}=\Delta_{N}, \sum_{1}^{N} z_{i}=z_{N}, W_{m}\left(R^{3 N}\right)=W_{m}, L_{s}^{2}\left(R^{3 N}\right)=L_{s}^{2}, G(\theta)=\prod_{1}^{N} f_{i}(\theta), f_{i}(\theta)=\left[r_{i}(\theta) / r_{i}\right]^{1 / 2}$, $i=1, \ldots, N$, a simple tensor product argument yields:

Proposition 3.3. Let $H_{0}(\theta)$ be the operator in $L^{2}$ defined as the closure of the tensor product $h_{0}(\theta) \otimes I \ldots \otimes I+I \otimes h_{0}(\theta) \otimes \ldots \otimes I+\ldots+I \otimes \ldots \otimes h_{0}(\theta)$ where $h_{0}(\theta)$ is the operator in $L^{2}\left(R^{3}\right)$ of Lemma 2.3. Let $\Delta_{N}(\theta)=U(\theta) \Delta_{N} U(\theta)^{-1}$ be the unitary image of the Laplace operator $\Delta_{N}$ under $U(\theta), \theta \in R$. Then:

(i) For any $\varepsilon, 0<\varepsilon<\pi / 2$, there is $\delta>0$ such that $H_{0}(\theta)$ has domain $W_{2}$ and is a holomorphic family of type $A$ of $m$-sectorial operators with opening angle not exceeding $2 \varepsilon$ for all complex $\theta$ such that $|\theta|<\delta$. The quadratic form of $H_{0}(\theta)$ is $W_{1}$.

(ii) If $\theta \in R$ the following operator identity holds:

$$
-\Delta_{N}(\theta)=G(\theta) H_{0}(\theta) G(\theta)^{-1}
$$

(iii) The operator $-\Delta_{N}(\theta)$ can be continued to a holomorphic operator family in $L^{2}$ for all $\theta$ in $|\theta|<\delta$, and $\sigma\left(\Delta_{N}(\theta)\right)=\sigma\left(H_{0}(\theta)\right)$.

Proposition 3.4. Let $H_{0}(\gamma, \theta)$ be the operator family in $L^{2}$ defined as the closure of the operator $H_{0}^{\prime}(\gamma, \theta)=H_{0}(\theta)+\gamma \sum_{1}^{N}\left(\alpha z_{i}+\beta r_{i}\right)$ with domain $\bigotimes_{1}^{N}\left(W_{2}\left(R^{3}\right) \cap L_{1}^{2}\left(R^{3}\right)\right)$. Then $D\left(H_{0}(\gamma, \theta)\right)=W_{2} \cap L_{1}^{2}$, and Theorem 2.6, Corollary 2.10 remain unchanged under the replacements $h_{0}(\gamma, \theta) \rightarrow H_{0}(\gamma, \theta), W_{m}\left(R^{3}\right) \rightarrow W_{m}, L_{s}^{2}\left(R^{3}\right) \rightarrow L_{s}^{2},-\Delta+\gamma z \rightarrow$ $-\Delta_{N}+\gamma z_{N}, h_{0}(\theta) \rightarrow H_{0}(\theta),-\Delta+F z \rightarrow-\Delta_{N}+F z_{N}$.

Proof. $H_{0}^{\prime}(\gamma, \theta)$ is closable because $h_{0}(\gamma, \theta)$ is a closed operator in $L^{2}\left(R^{3}\right)$ and

$$
H_{0}^{\prime}(\gamma, \theta)=h_{0}(\gamma, \theta) \otimes I \otimes \ldots \otimes I+\ldots+I \otimes I \otimes \ldots \otimes h_{0}(\gamma, \theta)
$$


Proceeding exactly as in Lemmas 2.8 and 2.9 (up to some simple modifications to be omitted for brevity) one has the further quadratic estimate

$$
\left\|\left\{H_{0}^{\prime}(\theta)+\gamma \sum_{1}^{N}\left(\alpha z_{i}+\beta r_{i}\right)\right\} \psi\right\|^{2}+b\|\psi\|^{2} \geqq a\left\{\left\|H_{0}^{\prime}(\theta) \psi\right\|^{2}+c\left\|\gamma \sum_{1}^{N} r_{i} \psi\right\|^{2}\right\}
$$

valid for any $\psi \in \bigotimes_{1}^{N}\left(W_{2}\left(R^{3}\right) \cap L_{1}^{2}\left(R^{3}\right)\right)$ under the same conditions and with the same uniformities as in Lemma 2.9. Since $\bigotimes_{1}^{N}\left(W_{2}\left(R^{3}\right) \cap L_{1}^{2}\left(R^{3}\right)\right) \cong W_{2} \cap L_{1}^{2}$, a standard closure argument shows that $H_{0}(\gamma, \theta)$ has domain $W_{2} \cap L_{1}^{2}$. To prove the remaining part of the theorem, first remark that, by (3.5) and the fact that $N$ $\bigotimes\left(W_{2}\left(R^{3}\right) \cap L_{1}^{2}\left(R^{3}\right)\right)$ is a core of $H_{0}(\gamma, \theta)$, we can replace $h_{0}(\gamma, \theta)$ by $H_{0}(\gamma, \theta)$ in Lemma 2.7. Now, proceeding as in the proof of Theorem 2.6, taking the form sum of $H_{0}(\theta)$ and $\sum_{1}^{N}\left(\alpha z_{i}+\beta r_{i}\right)$ and applying the quadratic estimate (3.5) we conclude that $H_{0}(\gamma, \theta)$ is $m$-sectorial. Again as in Theorem 2.6, we can use the quadratic estimate (3.5) and the compactness of $\left(-\Delta_{N}+\sum_{1}^{N} r_{i}+1\right)^{-1}$ to prove the compactness of $\left[H_{0}(\gamma, \theta)-E\right]^{-1}$ by the closed graph theorem. The strong convergence statements of Theorem 2.6(ii) are proved in the present case by exactly the same argument because $h_{0}(\gamma, \theta)$ can be replaced by $H_{0}(\gamma, \theta)$ in Lemma 2.7, and the same is true for the assertions of Corollary 2.10 just by remarking that the dilation analytic vectors, both in the canonical sense as well as in the parabolic one, are now intended in $L^{2}\left(R^{3 N}\right)$. This proves Proposition 3.4.

Let us now specify our assumptions on the interparticle potentials.

Assumptions 3.5. Let $V_{0}: R^{3} \rightarrow R$ be an arbitrary two-body potential $V_{i}\left(\mathbf{r}_{i}\right)$ or $V_{i j}\left(\mathbf{r}_{i}-\mathbf{r}_{j}\right), i, j=1, \ldots, N$. Then let us assume

(i) $V_{0}$ belongs to the class $C_{\alpha}$ of dilation analytic potentials (see e.g. [10, XIII.10]). This means, let us recall, that the multiplication by $V_{0}$ is compact if considered as an operator from $W_{2}\left(R^{3}\right)$ to $L^{2}\left(R^{3}\right)$, and that $V_{0}(\phi) \equiv V_{0}\left(e^{\phi} x, e^{\phi} y, e^{\phi} z\right): W_{2}\left(R^{3}\right) \rightarrow L^{2}\left(R^{3}\right)$ admits a compact valued analytic continuation from $R$ to the strip $|\operatorname{Im} \phi|<\alpha$.

(ii) If $V_{0}=V_{i}$, for any $\phi$ in $|\operatorname{Im} \phi|<\alpha V_{0}(\phi)$ is dilation analytic with respect to the transformation $t(\theta)$ for $|\theta|<\delta$, i.e. the multiplication operator from $W_{2}\left(R^{3}\right)$ to $L^{2}\left(R^{3}\right): t(\theta) V_{0}(\phi) t(\theta)^{-1} \equiv V_{0}(\theta, \phi) \equiv V_{0}\left(e^{(\theta+\phi)} x, e^{(\theta+\phi)} y, e^{\phi}\left(1-e^{\theta}\right) r / 2+e^{\phi}\left(1+e^{\theta}\right) z / 2\right)$ admits a compact operator valued analytic continuation from $\theta \in R$ to $\Theta_{\delta}=\{\theta|| \theta \mid<\delta\}$. If $V_{0}=V_{i j}$, for any $\phi$ as above $V_{0}(\phi)$ is dilation analytic with respect to the transformation $U(\theta)$ for $|\theta|<\delta$, i.e. the multiplication operator from $W_{2}$ to $L^{2} U(\theta) V_{0}(\phi) U(\theta)^{-1}$ $\equiv V_{0}(\theta, \phi) \equiv V_{0}\left(e^{(\theta+\phi)} x, e^{(\theta+\phi)} y, e^{\phi}\left(1-e^{\theta}\right) r^{\prime} / 2+e^{\phi}\left(1+e^{\theta}\right) z / 2\right), \quad r^{\prime} \equiv r_{i}-r_{j}$, admits $a$ bounded-operator valued analytic continuation from $\theta \in R$ to $\Theta_{\delta}=\{\theta|| \theta \mid<\delta\}$.

(iii) $V_{i}, V_{i j}$ are such that $H(F)$ is essentially self-adjoint on $C_{0}^{\infty}\left(R^{3 N}\right)$.

Remarks. (a) The class of two-body potentials satisfying the above assumptions is not empty. For instance it includes the Yukawa and the Coulomb ones, which yield the essential self-adjointness of $H(F)$ on $C_{0}^{\infty}\left(R^{3 N}\right)$ (see e.g. $[10$, X.5]). 
(b) Let $V(\theta, \phi)=\sum_{1}^{N} V_{i}(\theta, \phi)=\sum_{i<j}^{N} V_{i j}(\theta, \phi)$. Then $V$ is bounded as a map from $W_{2}$ to $L^{2}$ and, a fortiori, bounded as a map from $W_{2} \cap D\left(z_{N}\right)$ and from $W_{2} \cap L_{1}^{2}$ to $L^{2}$ for all $(\theta, \phi)$ in $\Theta_{\delta} \times(-\alpha, \alpha)$.

We are now in position to define the two operator families in $L^{2}$ associated with $H(F)$ :

$$
\begin{aligned}
H(F, \phi)= & -e^{-2 \phi} \Delta_{N}+V(\phi)+e^{\phi} F z_{N}, \quad D(H(F, \phi))=W_{2} \cap D\left(z_{N}\right), \\
H(F, \theta, \phi)= & e^{-2 \phi} H_{0}\left(e^{3 \phi} F, \theta\right)+V(\theta, \phi) \\
= & e^{-2 \phi} H_{0}(\theta)+V(\theta, \phi)+e^{\phi} F \sum_{1}^{N}\left(\alpha z_{i}+\beta r_{i}\right), \\
& D(H(F, \theta, \phi))=W_{2} \cap L_{1}^{2} .
\end{aligned}
$$

The relevant properties of these operator families are summarized in the following result.

Theorem 3.6. (i) If $F$ and $\phi$ are real $H(F, \phi)$ is essentially self-adjoint.

(ii) If $F$ and $\phi$ are complex, $0<\arg (F)+3 \operatorname{Im} \phi<\pi, H(F, \phi)$ is a holomorphic family of type $A$ (in $F$ at fixed $\phi$, and in $\phi$ at fixed $F$ ).

(iii) If $F, \theta, \phi$ are complex, $\theta \in M, 0<\arg (F)+3 \operatorname{Im} \phi<\pi, H(F, \theta, \phi)$ is a holomorphic family of type $A$ (in each single variable, the remaining two being held fixed) of $m$-sectorial operators with compact resolvents.

(iv) If $F$ is real, $\operatorname{Im} \phi>0$, there is $E_{0}>-\infty$ such that $[H(F, \phi)-E]^{-1}$ converges strongly to $\left[\bar{H}\left(F, \phi_{0}\right)-E\right]^{-1}$ when $\operatorname{Im} \phi \rightarrow 0, \operatorname{Re} \phi=\phi_{0}$, uniformly on compacts in $E$ for $\operatorname{Re} E<E_{0}, \operatorname{Im} E>0$.

(v) If $F$ is real, $\operatorname{Im} \phi=\pi / 6$, there is $E_{0}>-\infty$ such that $[H(F, \theta, \phi)-E]^{-1}$ converges strongly to $[H(F, \phi)-E]^{-1}$ when $\operatorname{Re} \theta=0, \operatorname{Im} \theta \rightarrow 0$, uniformly on compacts in $E$ as above.

Proof. (i) $H(F, \phi)$ is essentially self-adjoint on $C_{0}^{\infty}$ by Assumption 3.5 (iii) and the unitary equivalence. Since $W_{2} \cap L_{1}^{2} \supset C_{0}^{\infty}, H(F, \phi)$ has the same closure and this proves (i).

(ii) We have of course $H(F, \phi)=T_{0}(F, \phi)+V(\phi)$, where $T_{0}(F, \phi)$ is by Lemma 3.1 a holomorphic family with the stated properties. By our assumptions $V(\phi)$ is bounded relative to $T_{0}(F, \phi)$ with relative bound zero because $D\left(T_{0}(F, \phi)\right)$ $=W_{2} \cap D\left(z_{N}\right)$. Hence by a standard perturbation result $H(F, \phi)$ is closed and since $H(F, \phi) u$ is of course a holomorphic vector function of $(F, \phi)$ for any $u \in W_{2} \cap D\left(z_{N}\right)$, $H(F, \phi)$ is a holomorphic family of type $A$ by definition.

(iii) is proved exactly as (ii) because of the properties of $H_{0}(\gamma, \theta)\left(\right.$ with $\left.\gamma=F e^{3 \phi}\right)$ and $V(\theta, \phi)$. Remark that the compactness of the resolvent is a consequence of the compactness of $\left[H_{0}\left(F e^{3 \phi}, \theta\right)-E\right]^{-1}$ and the relative boundedness of $V(\theta, \phi)$ with respect to $H_{0}\left(F e^{3 \phi}, \theta\right)$ with relative bound zero, in view of a well known result (see e.g. [9, Theorem IV.3.17]).

(iv) The numerical range of $e^{3 \phi} z_{N}$ is the straight line through the origin with angular coefficient $3 \operatorname{Im} \phi$. Since $V(\phi)$ is relatively bounded with respect to $-\Delta_{N}$ with relative bound zero, given $\varepsilon>0$ we can always find $E_{0}<0$ independent of $\varepsilon$ such that for $0<\operatorname{Im} \phi<\varepsilon$ the numerical range of $-\Delta_{N}+e^{2 \phi} V(\phi)$ is contained in a 
sector of arbitrarily small opening angle and vertex $E_{0}$ about the real axis. Hence the region: $\left\{E|\operatorname{Im} E>0| \operatorname{Re} E<E_{0}\right\}$ has positive distance from the union over $\varepsilon>\operatorname{Im} \phi>0$ of $W\left(e^{2 \phi} H(F, \phi)\right)$ and therefore $\left[e^{2 \phi} H(F, \phi)-E\right]^{-1}$ is bounded uniformly over $\operatorname{Im} \phi>0$. Hence it converges strongly to $\left[e^{2 \phi_{0}} \bar{H}\left(F, \phi_{0}\right)-E\right]^{-1}$ with the stated uniformity because $e^{2 \phi} H(F, \phi) u \rightarrow e^{2 \phi_{0}} H\left(F, \phi_{0}\right) u, \phi \rightarrow \phi_{0}$, for all $u \in C_{0}^{\infty}$ which is a core of $\bar{H}\left(F, \phi_{0}\right)$.

(v) Again, given $\varepsilon>0$ we have to control $W\left(e^{2 \phi} H(F, \theta, \phi)\right)$ uniformly for $\operatorname{Re} \theta=0,0<\operatorname{Im} \theta<\varepsilon, \operatorname{Im} \phi=\pi / 6$. To do this, first remark that for $\operatorname{Im} \phi=\pi / 6$ the numerical range of $F e^{3 \phi} \sum_{1}^{N}\left(\alpha z_{i}+\beta r_{i}\right)$ lies in $\{E \mid \operatorname{Re} E \geqq 0\}$. Now $e^{2 \phi} H(F, \theta, \phi)$ $=H_{0}(\theta)+e^{2 \phi} V(\theta, \phi)+F e^{3 \phi} \sum_{1}^{N}\left(\alpha z_{i}+\beta r_{i}\right)$ and the numerical range of $H_{0}(\theta)$ $+e^{2 \phi} V(\theta, \phi)$ can be controlled exactly as above by the strict sectoriality (with arbitrarily small opening angle) of $H_{0}(\theta)$ and the relative boundedness (with relative bound zero) of $V$ with respect to $H_{0}(\theta)$. Hence, again, the assertion follows from the strong convergence $H(F, \theta, \phi) u \rightarrow H(F, \phi) u$ as $\operatorname{Im} \theta \rightarrow 0, \operatorname{Re} \theta=0$, taking place for any $u \in W_{2} \cap L_{1}^{2}$. This concludes the proof of Theorem 3.6.

Let us now verify that the eigenvalues of $H(F, \theta, \phi)$ are resonances of $\bar{H}(F)$ according to the standard notion of this concept (see e.g. [10, Sects. XII.6 and XIII.10]).

Theorem 3.7. Let $F$ be real, $\theta$ and $\phi$ complex, $\theta \in M, 0<\operatorname{Im} \phi<\pi$. Then:

(i) The eigenvalues of $H(F, \theta, \phi)$, together with their (algebraic) multiplicities, are independent of $(\theta, \phi)$.

(ii) All eigenvalues of $H(F, \theta, \phi)$ lie in $\{E \mid \operatorname{Im} E \leqq 0\}$ and are resonances of $\bar{H}(F)$ in the following sense: if $\psi_{1}, \psi_{2}$ are jointly canonical and parabolic dilation analytic vectors for $(\theta, \operatorname{Im} \phi) \in \Theta_{\delta} \times(-\alpha, \alpha)$ then $f_{1,2}(E)=\left\langle\psi_{1},(\bar{H}(F)-E)^{-1} \psi_{2}\right\rangle$ has a meromorphic continuation from $\operatorname{Im} E>0$ to the whole complex plane. The only poles possible are at the eigenvalues of $H(F, \theta, \phi)$. The set of singularities $\left\{E \mid f_{1,2}\right.$ has a pole at $E$ for some $\left.\left(\psi_{1}, \psi_{2}\right)\right\}$ coincides with $\sigma(H(F, \theta, \phi))$.

(iii) If $E \in \sigma(H(F, \theta, \phi)) \cap R$ then $E$ is an eigenvalue of $\bar{H}(F)$.

(iv) $\bar{H}(F)$ has no singular continuous spectrum. $\sigma_{p p}(\bar{H}(F))$ has no finite accumulation point.

Proof. The argument goes as in Corollary 2.10 and depends on the identity

$$
\left[G(\theta) H(F, \theta, \phi) G(\theta)^{-1}-E\right]^{-1}=G(\theta)[H(F, \theta, \phi)-E]^{-1} G(\theta)^{-1}
$$

which can be proved exactly as in Lemmas 2.3 and 2.4. (3.8) yields indeed the independence of $\theta$ of the spectrum through the usual arguments. In addition the dilation analyticity in $\phi$ for any fixed $\theta$ makes the spectrum independent also on $\phi$. Thus (i) holds and (ii) is an elementary consequence of (iv) and (v) of Theorem 3.6, because the dilation analyticity, first in $\theta$ and then in $\phi$, makes the scalar products $\left\langle\psi_{1}(\bar{\theta}, \bar{\phi}), G(\theta)[H(F, \theta, \phi)-E]^{-1} G(\theta)^{-1} \psi_{2}(\theta, \phi)\right\rangle$ independent of $(\theta, \phi)$ over $\theta \in M$, $0<\operatorname{Im} \phi<\pi$. [Remark that $G(\theta), G(\theta)^{-1} \rightarrow 1$ as $\operatorname{Im} \theta \rightarrow 0, \operatorname{Re} \theta=0$.] Finally (iii) and (iv) are well known consequences of the dilation analyticity, given the spectral nature of $H(F, \theta, \phi)$. This proves Theorem 3.7. 
Remark. We do not attempt to prove the physically reasonable fact (and rigorously proved in the two-body case: see e.g. [10, XIII.4]) that $\sigma(\bar{H}(F))=\sigma_{a c}(\bar{H}(F))$ $=(-\infty,+\infty)$. We limit ourselves to remark that $\sigma(\bar{H}(F))=(-\infty,+\infty)$. For, it is an immediate consequence of the above result that if $\sigma(\bar{H}(F)) \neq(-\infty,+\infty)$ then it consists entirely of isolated eigenvalues of finite multiplicity, accumulating possibly only at infinity. Then $[\bar{H}(F)-E]^{-1}$ would be compact and this is impossible.

\section{Stability and Borel Summability}

The Stark effect can be described by saying that the bound states of any atomic system turn into resonances as soon as the electric field is turned on. We proceed now to give a proof of this fact at least for the eigenvalues of the $N$-body Schrödinger operator $-\Delta_{N}+V\left(-\Delta_{N}=\sum_{1}^{N} \Delta_{i}, V=\sum_{1}^{N} V_{i}+\sum_{i<j}^{N} V_{i j}\right)$ lying below all thresholds. Moreover, if any such eigenvalue is simple, this stability assertion will be strengthened to the Borel summability of its perturbation expansion to the nearby resonance. The present stability result reads as a direct generalization of Theorem III. 2 of [5].

Theorem 4.1. Let $H(0)$ be the Schrödinger operator in $L^{2}$ defined by $-\Delta_{N}+V$ on $W_{2}$, where any two-body potential satisfies assumptions 3.5 so that $H(0)$ is selfadjoint and bounded below. Let $E_{0}$ be an eigenvalue of $H(0)$ of multiplicity j lying below all thresholds. Then for $(\theta, \phi)$ complex, $\theta \in M, 0<\operatorname{Im} \phi<\pi / 3, F>0$, the operator $H(F, \theta, \phi)$ has exactly jeigenvalues (counted according their algebraic multiplicity) near $E_{0}$ for $F$ small. These eigenvalue converge to $E_{0}$ as $F \rightarrow 0$.

The proof is to be obtained through four preliminary lemmas. The basic argument consists in taking advantage of the uniform strict sectoriality of $H(F, \theta, \phi)$ as well as of any cluster decomposition of it in order to obtain uniform bounds on the resolvents for $|F|$ small and $E$ near $E_{0}$ through the Weinberg-Van Winter equation.

Let us first reobtain within the present formalism Herbst's stability result in the two-body case $(N=1)$.

Lemma 4.2. Let the multiplication operator $V: R^{3} \rightarrow R$ be dilation analytic for $(\theta, \operatorname{Im} \phi) \in \Theta_{\delta} \times(-\alpha, \alpha)$, and define in $L^{2}\left(R^{3}\right)$ the following operators:

$$
\begin{aligned}
& k(F, \theta, \phi, E)=V(\theta, \phi)\left(h_{0}(F, \theta, \phi)-E\right)^{-1}, \quad k(\theta, \phi, E)=V(\theta, \phi)\left(h_{0}(\theta, \phi)-E\right)^{-1} \\
& \left.H(F, \theta, \phi)\right|_{N=1} \equiv h(F, \theta, \phi)=h_{0}(F, \theta, \phi)+V(\theta, \phi), \quad D(h(\cdot))=W_{2}\left(R^{3}\right) \cap L_{1}^{2}\left(R^{3}\right)
\end{aligned}
$$

with $|F|>0,0<\arg (F)+3 \operatorname{Im} \phi<\pi, \theta \in M$, where $h_{0}(\theta, \phi)=e^{-2 \phi} h_{0}(\theta), h_{0}(F, \theta, \phi)$ $=e^{-2 \phi} h_{0}\left(e^{3 \phi} F, \theta\right)$. Then $\|k(F, \cdot)-k(\cdot)\| \rightarrow 0$ as $|F| \rightarrow 0$, uniformly on compacts with respect to $(\theta, \phi, E)$ for $(\theta, \operatorname{Im} \phi) \in M \times(-\alpha, \alpha)$ and $d(E, \theta, \phi)>0$, where $d(E, \theta, \phi)$ is the distance between $E$ and the union over $|F| \geqq 0$ of $W\left(h_{0}(F, \cdot)\right)$.

Proof. By exactly the same argument of [5, Proposition III.1(ii)], this norm convergence holds if $h_{0}(F, \cdot), h_{0}(\cdot)$ are replaced by $e^{-2 \phi}\left(-\Delta+e^{3 \phi} F(\alpha z+\beta r)\right)$ and 
$-e^{-2 \phi} \Delta$, respectively [remark in this connection that $\left[\left(-e^{-2 \phi} \Delta-E\right)^{-1}, \alpha z+\beta r\right]$ $\left.=2 i e^{-2 \phi}\left(-e^{-2 \varphi} \Delta-E\right)^{-1}\left(\alpha p_{z}+\beta p_{r}\right)\left(-e^{-2 \phi} \Delta-E\right)^{-1}\right]$. Write:

$$
\begin{aligned}
\left(h_{0}(\cdot)-E\right)^{-1} \equiv & \left(-e^{-2 \phi} \Delta+1\right)^{-1}\left(-e^{-2 \phi} \Delta+1\right)\left(h_{0}(\cdot)-E\right)^{-1} \\
\left(h_{0}(F, \cdot)-E\right)^{-1} \equiv & \left(-e^{-2 \phi} \Delta+e^{\phi} F(\alpha z+\beta r)+1\right)^{-1}\left(-e^{-2 \phi} \Delta+e^{\phi} F(\alpha z+\beta r)+1\right) \\
& \cdot\left(h_{0}(F, \cdot)-E\right)^{-1} .
\end{aligned}
$$

By (2.8) and (ii) of Theorem 2.6, $\left(-e^{-2 \phi} \Delta+e^{\phi} F(\alpha z+\beta r)+1\right)\left(h_{0}(F, \cdot)-E\right)^{-1}$ is uniformly bounded over $|F|>0$ and converges strongly to the bounded operator $\left(-e^{-2 \phi} \Delta+1\right)\left(h_{0}(\cdot)-E\right)^{-1}$ as $|F| \rightarrow 0$. Since the norm convergence

$$
\left\|V(\cdot)\left(-e^{-2 \phi} \Delta+e^{\phi} F(\alpha z+\beta r)-E\right)^{-1}-V(\cdot)\left(-e^{-2 \phi} \Delta-E\right)^{-1}\right\| \rightarrow 0
$$

as $|F| \rightarrow 0$ takes place between compact operators, the lemma is proved.

Lemma 4.3. Theorem 4.1 holds for the two-body case $N=1$.

Proof. If $E_{0}<0$ is an isolated eigenvalue of $h(0,0,0)=-\Delta+V$, it is an isolated eigenvalue also of $h(0,0, \phi)=-e^{-2 \phi} \Delta+V(\phi)$ for $|\operatorname{Im} \phi|<\alpha$. By the identity $\left(f(\theta) h(0, \theta, \phi) f(\theta)^{-1}-E\right)^{-1} \equiv f(\theta)(h(0, \theta, \phi)-E)^{-1} f(\theta)^{-1}$, the analyticity in $\theta$ and the unitarity of $t(\theta)$ for $\theta$ real, $E_{0}$ is an isolated eigenvalue, with the same (algebraic) multiplicity also of $h(0, \theta, \phi)$ for $(\theta, \operatorname{Im} \phi)$ in $\Theta_{\delta} \times(-\alpha, \alpha)$. Hence, by Lemma 4.1, the assertion follows by exactly the same argument of Herbst [5, Theorem III.3], if we replace $K(\varepsilon, \theta, z), K(\theta, z), H(\varepsilon, \theta), H_{0}(\varepsilon, \theta)$ by $k(F, \theta, \phi, E), k(\theta, \phi, E), h(F, \theta, \phi)$, $h_{0}(F, \theta, \phi)$, respectively.

Remark. Let $E_{0}<0$ belong to the open set $\varrho(h(0,0,0))$. If as usual $E_{0}$ is interpreted as an eigenvalue of multiplicity 0 , the above result implies that $E_{0} \in \varrho(h(F, \theta, \phi))$ for $|F|$ suitably small, and that $(h(F, \theta, \phi)-E)^{-1}$ is bounded uniformly with respect to $F$ for all $E$ such that $\left|E-E_{0}\right|$ is small (depending on $F$ ).

Let us now introduce the Weinberg disconnected and connected kernels $D(F, \theta, \phi, E), D(\theta, \phi, E), I(F, \theta, \phi, E), I(\theta, \phi, E)$ corresponding to $(H(F, \theta, \phi)-E)^{-1}$, $(H(0, \theta, \phi)-E)^{-1}$, respectively, defined as usual as the sum of all disconnected and, respectively, barely connected diagrams in the geometric expansions:

$$
\begin{aligned}
& (H(F, \theta, \phi)-E)^{-1}=\left(H_{0}(F, \theta, \phi)-E\right)^{-1}\left\{1+V(\cdot)\left(H_{0}(F, \theta, \phi)-E\right)^{-1}\right\}^{-1}, \\
& (H(0, \theta, \phi)-E)^{-1}=\left(H_{0}(0, \theta, \phi)-E\right)^{-1}\left\{1+V(\cdot)\left(H_{0}(0, \theta, \phi)-E\right)^{-1}\right\}^{-1}
\end{aligned}
$$

for all values of $E$ for which these expansions converge. (For these, and several other notions freely used in what follows without further reference, see $[10$, XIII.5].) Remark that under our assumptions on the potentials there is a convergence region independent of $F$ as long as $\theta \in M, 0<\arg (F)+3 \operatorname{Im} \phi<\pi$.

Lemma 4.4. Let $F, \theta, \phi$ be as in Theorem 4.1. Let $G(F, \theta, \phi, E)$ be the operator corresponding to an arbitrary diagram in the geometric expansion (4.1), and $G(\theta, \phi, E)$ the operator corresponding to the same diagram in the geometric expansion (4.2). Then there is $E^{\prime}$ such that, uniformly on compacts in $\left\{E\left|\operatorname{Re} E<E^{\prime}\right| \operatorname{Im} E>0\right\}$ and in 
$(\theta, \phi)$ as above one has:

(i) $G(F, \cdot, E)$ is compact.

(ii) If the diagram is connected, $G(\cdot, E)$ is compact.

(iii) If the diagram is disconnected, $G(F, \cdot, E) \rightarrow \vec{s} G(\cdot, E)$ as $|F| \rightarrow 0$.

(iv) If the diagram is barely connected, $G(F, \cdot, E) \rightarrow G(\cdot, E)$ in norm as $|F| \rightarrow 0$.

Proof. Let $V_{1}(\cdot)$ be an arbitrary two-body potential satisfying (3.5). Then $V_{1}(\cdot): W_{2} \rightarrow L^{2}$ is bounded, and the immersion of $W_{2} \cap L_{1}^{2}$ in $L^{2}$ is compact. Thus it is easy to see that $V_{1}(\cdot): W_{2} \cap L_{1}^{2} \rightarrow L^{2}$ is compact. Since $D(H(F, \cdot))=W_{2} \cap L_{1}^{2}$, and $\sigma\left(H_{0}(F, \cdot)\right)=\emptyset, V_{1}(\cdot)\left(H_{0}(F, \cdot)-E\right)^{-1}$ is compact for all $E$. Thus (i) is proved for all $E$ since one has $G(F, \cdot, E)=R(F, \cdot E) V_{1}(\cdot) G_{1}(\cdot)$ where $R(F, \cdot, E)=\left(H_{0}(F, \cdot)-E\right)^{-1}$ and $G_{1}(\cdot)$ is bounded for all $E$ with the stated uniformities. (iii) is trivial since $R(F, \cdot, E) \rightarrow R(0, \cdot, E)=\left(H_{0}(0, \cdot)-E\right)^{-1}$ as $|F| \rightarrow 0$ by Theorem 3.6(v). (ii) holds if $G(\cdot, E)=R(0, \cdot, E) V_{1}(\cdot) \ldots R(0, \cdot, E) V_{N}(\cdot)$ is compact for $V_{1} \neq V_{2} \neq \ldots \neq V_{N}$. The identity $R(0, \cdot, E)=G(\theta)\left(-e^{-2 \phi} \Delta_{N}(\theta)-E\right)^{-1} G(\theta)^{-1}, \theta \in R,|\operatorname{Im} \phi|<\alpha, \Delta_{N}(\theta)=U(\theta) \Delta_{N} U(\theta)^{-1}$, yields $G(\theta, \phi, E)=G(\theta)\left(-e^{-2 \phi} \Delta_{N}(\theta)-E\right)^{-1} V_{1}(\theta, \phi) \ldots\left(-e^{-2 \phi} \Delta_{N}(\theta)-E\right)^{-1} V_{N}(\theta, \phi) G(\theta)^{-1}$ which is compact for $\theta \in R$ by the unitary equivalence of

$$
\left(-e^{-2 \phi} \Delta_{N}(\theta)-E\right)^{-1} V_{1}(\theta, \phi) \ldots\left(-e^{-2 \phi} \Delta_{N}(\theta)-E\right)^{-1} V_{N}(\theta, \phi)
$$

with the compact operator $G(0, \phi, E)$. Since $G(\theta, \phi, E)$ is holomorphic for $\theta \in \Theta_{\delta}$ when $|\operatorname{Im} \phi|<\alpha$, the assertion follows by a known result [10, XIII.5]. To show (iv), consider first the $N=2$ case. Since $R(F, \cdot, E) \rightarrow R(0, \cdot, E)$, we have to prove only the norm convergence, with the stated uniformities, of the compact operator $K(F, F)=R(F, \cdot, E) V_{1}(\cdot) R(F, \cdot, E) V_{2}(\cdot)$ to $K(0,0)=R(0, \cdot, E) V_{1}(\cdot) R(0, \cdot, E) V_{2}(\cdot)$ which is also compact. Write $K(F, F)-K(0,0)=K(F, F)-K(F, 0)+K(F, 0)-K(0,0)$, with obvious meaning of $K(F, 0)$. Since $R(F, \cdot, E)=R(F, \cdot, E)\left(H_{0}(0, \cdot)-E\right) R(0, \cdot, E)$, $\|K(F, 0)-K(0,0)\| \rightarrow 0$ as $|F| \rightarrow 0$ with the stated uniformities because $K(0,0)$ is compact and $R(F, \cdot, E)\left(H_{0}(0, \cdot)-E\right) \rightarrow I$. To see that $\|K(F, F)-K(F, 0)\| \rightarrow 0$, by the uniform boundedness of $R(F, \cdot, E)\left(H_{0}(0, \cdot)-E\right)$ it suffices $\|K(0, F)-K(0,0)\| \rightarrow 0$ or, taking the adjoints, $\left\|\bar{V}_{2}(\cdot) R(\bar{F}, \cdot, \bar{E}) \bar{V}_{1}(\cdot) R(0, \cdot, \bar{E})-\bar{V}_{2}(\cdot) R(0, \cdot, \bar{E}) \bar{V}_{1}(\cdot) R(0, \cdot, \bar{E})\right\|$ $=\left\|K(0, F)^{\dagger}-K(0,0)^{\dagger}\right\| \rightarrow 0$. Now for $|F| \geqq 0$ we can always write $H_{0}(F, \cdot)=H_{2}(F, \cdot)$ $\otimes I+I \otimes H_{2}^{\prime}(F, \cdot)$, where the tensor product refers to the decomposition of $L^{2}\left(R^{6}\right)$ induced by that of $R^{6}$ into the three coordinates of $V_{2}$ and three independent ones. Since $H_{2}(F, \cdot)$ and $H_{2}^{\prime}(F, \cdot)$ are $m$-sectorial, if we denote by $R_{2}(F, \cdot, E), R_{2}^{\prime}(F, \cdot, E)$ the corresponding resolvents we have (see Reed and Simon [11]):

$$
R(\bar{F}, \cdot \bar{E})=(2 \pi i)^{-1} \int_{\Gamma} R_{2}^{\prime}(\bar{F}, \cdot, \lambda) \otimes R_{2}(\bar{F}, \cdot, \bar{E}-\lambda) d \lambda
$$

$\Gamma$ being the straight line $\operatorname{Im}(\lambda-\bar{E} / 2)=\operatorname{tg} \gamma \operatorname{Re}(\lambda-\bar{E} / 2),-\gamma=\operatorname{Im} \phi+\arg (F)+\operatorname{Im} \theta / 2$. By the independence of the variables 2 and $2^{\prime}$ one easily shows that

$$
\begin{aligned}
& K(0, F)^{\dagger}=(2 \pi i)^{-1} \int_{\Gamma} \bar{V}_{2}(\cdot) R_{2}(0, \cdot, \bar{E}-\lambda) \otimes R_{2}^{\prime}(0, \cdot, \lambda) \bar{V}_{1}(\cdot) R(0, \cdot \bar{E}) d \lambda \\
& \quad+(2 \pi i)^{-1} \int_{\Gamma} B_{2}^{\prime}(\bar{F}, \bar{E}, \lambda)\left\{\bar{V}_{2}(\cdot) R_{2}(\bar{F}, \cdot, \bar{E}-\lambda) \otimes R_{2}^{\prime}(0, \cdot \lambda)-\bar{V}_{1}(\cdot) R_{2}(0, \cdot \bar{E}-\lambda)\right. \\
& \left.\quad \otimes R_{2}^{\prime}(0, \cdot, \lambda)\right\} \bar{V}_{1}(\cdot) R(0, \cdot, \bar{E}) d \lambda+(2 \pi i)^{-1} \int_{\Gamma}\left\{B_{2}^{\prime}(\bar{F}, \bar{E}, \lambda)-B_{2}^{\prime}(0, \bar{E}, \lambda)\right\} \\
& \quad \cdot\left\{\bar{V}_{2}(\cdot) R_{2}(0, \cdot, \bar{E}-\lambda) \otimes R_{2}^{\prime}(0, \cdot, \lambda) \bar{V}_{1}(\cdot) R(0, \cdot, \bar{E})\right\} d \lambda,
\end{aligned}
$$


where $B_{2}^{\prime}(\bar{F}, \bar{E}, \lambda)=R_{2}^{\prime}(\bar{F}, \cdot, \bar{E})\left(H_{2}^{\prime}(0, \cdot)-\lambda\right),|F| \geqq 0$. Now the first integral is just $K(0,0)^{\dagger}$, and the second one vanishes in norm as $|F| \rightarrow 0$ because $B_{2}^{\prime}(\bar{F}, \bar{E}, \lambda)$ is uniformly bounded and $\bar{V}_{2}(\cdot) R_{2}(\bar{F}, \cdot, \bar{E}-\lambda) \rightarrow \bar{V}_{2}(\cdot) R_{2}(0, \cdot, \bar{E}-\lambda)$ in norm, uniformly over $\Gamma$, by Lemma 4.2. The third integral vanishes as $|F| \rightarrow 0$ in norm since the integrand is norm convergent to 0 , uniformly over $\Gamma$, because $B_{2}^{\prime}(\bar{F}, \bar{E}, \lambda) \rightarrow B_{2}^{\prime}(\bar{F}, \bar{E}, \lambda)$ uniformly over $\Gamma$ and $\bar{V}_{2}(\cdot) R_{2}(0, \cdot, \bar{E}-\lambda) \otimes R_{2}^{\prime}(0, \cdot, \lambda) \bar{V}_{1}(\cdot) R(0, \cdot, \bar{E})$ is compact [this is evident for $\theta=0$ by the inequality $\left(p_{2}^{2}+1\right)^{-1}\left(\left(p_{2}^{\prime}\right)^{2}+1\right)^{-1} \leqq\left(p_{2}^{2}+\left(p_{2}^{\prime}\right)^{2}+1\right)^{-1}$ and extends by analyticity to all $\left.\theta \in \Theta_{\delta}\right]$. Consider now the $N=3$ case. Proceeding as above, it is enough to prove $\left\|K(0, F, F)^{\dagger}-K(0,0,0)^{\dagger}\right\| \rightarrow 0$ as $|F| \rightarrow 0$. For $|F| \geqq 0$ write $H_{0}(F, \cdot)=H_{23}(F, \cdot) \otimes I+I \otimes H_{23}^{\prime}(F, \cdot)$, where the tensor product refers to the decomposition of $L^{2}\left(R^{9}\right)$ according to the six coordinates in $V_{2}$ and $V_{3}$ and three independent ones. Then, in the same notation as above, we have:

$$
\begin{aligned}
K(0, F, F)^{\dagger}=(2 \pi i)^{-2} \int_{\Gamma} \int_{\Gamma} \bar{V}_{3}(\cdot) R_{23}(0, \cdot, \bar{E}-\lambda) \bar{V}_{2}(\cdot) R_{23}\left(0, \cdot, \bar{E}-\lambda^{\prime}\right) \\
\quad \otimes R_{23}^{\prime}(0, \cdot, \lambda) R_{23}^{\prime}\left(0, \cdot, \lambda^{\prime}\right) \bar{V}_{1}(\cdot) R(0, \cdot, \bar{E}) d \lambda d \lambda^{\prime} \\
\quad+(2 \pi i)^{-2} \int_{\Gamma} \int_{\Gamma}\left\{B_{23}^{\prime}\left(\bar{F}, \bar{E}, \lambda, \lambda^{\prime}\right) \bar{V}_{3}(\cdot) R_{23}(\bar{F}, \cdot, \bar{E}-\lambda) \bar{V}_{2}(\cdot) R_{23}\left(\bar{F}, \cdot, \bar{E}-\lambda^{\prime}\right)\right. \\
\left.\quad \otimes R_{23}^{\prime}(0, \cdot, \lambda) R_{23}^{\prime}\left(0, \cdot, \lambda^{\prime}\right) \bar{V}_{1}(\cdot) R(0, \cdot, \bar{E})\right\} d \lambda d \lambda^{\prime}+(2 \pi i)^{-2} \iint_{\Gamma}\left\{B_{23}^{\prime}\left(\bar{F}, \bar{E}, \lambda, \lambda^{\prime}\right)\right. \\
\left.-B\left(0, \bar{E}, \lambda, \lambda^{\prime}\right)\right\} \bar{V}_{3}(\cdot) R_{23}(0, \cdot, \bar{E}-\lambda) \bar{V}_{2}(\cdot) R_{23}(0, \cdot, \bar{E}-\lambda) \\
\quad \otimes R_{23}^{\prime}(0, \cdot, \lambda) R_{23}^{\prime}\left(0, \cdot, \lambda^{\prime}\right) \bar{V}_{1}(\cdot) R(0, \cdot, \bar{E}) d \lambda d \lambda^{\prime} .
\end{aligned}
$$

As above, the first term is $K(0,0,0)^{\dagger}$. The second term vanishes in norm as $|F| \rightarrow 0$ by the uniform boundedness of

$$
B_{23}^{\prime}\left(\bar{F}, \bar{E}, \lambda, \lambda^{\prime}\right)=R_{23}^{\prime}\left(F, \lambda^{\prime}\right)\left(H_{23}^{\prime}(0, \cdot)-\lambda^{\prime}\right)\left(H_{23}^{\prime}(0, \cdot)-\lambda\right)
$$

and the norm convergence valid for $N=2$; the same is true for the third one since $B_{23}^{\prime}\left(\bar{F}, \bar{E}, \lambda, \lambda^{\prime}\right) \rightarrow I$ on a compact. Hence the result is proved for $N=3$ and there is no difficulty to extend the proof to all $N$ by iterating the argument.

Notice in this connection that the independent coordinates needed in the above tensor product argument have to be chosen after performing the dilation $\left(x_{i}, y_{i}, z_{i}\right) \rightarrow\left(e^{\theta / 2} x_{i}, e^{\theta / 2} y_{i}, \alpha z_{i}+\beta r_{i}\right)$ which is to be kept fixed. Remark also that, strictly speaking, when at least one potential is a $V_{i j}$, the above decomposition is just a sum of two commuting operators without tensor products. Consequently the above argument holds if the tensor product is replaced by the ordinary operator product. This concludes the proof of Lemma 4.4.

Lemma 4.5. Let $F, \theta, \phi$ be as in Theorem 4.1. Then there is $E^{\prime}<0$ such that, if $S=\left\{E\left|\operatorname{Re} E<E^{\prime}\right| \operatorname{Im} E>0\right\}$, uniformly on compacts in $(F, \theta, \phi)$ one has:

(i) $D(F, \theta, \phi, E), I(F, \theta, \phi, E)$ are compact-operator valued holomorphic functions of $E$ from $S$ to $L^{2}$, and compact-operator valued meromorphic functions from $C$ to $L^{2}$.

(ii) $D(\theta, \phi, E)$ is a bounded-operator valued holomorphic function of $E$ from $S$ to $L^{2}$, and $I(\theta, \phi, E)$ is a compact-operator valued holomorphic function of $E$ from $S$ to $L^{2}$.

(iii) $A s|F| \rightarrow 0, D(F, \theta, \phi, E)_{\rightarrow} D(\theta, \phi, E), I(F, \theta, \phi, E) \rightarrow I(\theta, \phi, E)$ in norm, uniformly with respect to $E$ on compacts in $S$. 
Proof. As in Theorem 3.6 one easily shows that there is $E^{\prime}<0$ such that the union over $|F| \geqq 0$ of the numerical ranges of $H(F, \theta, \phi)$ has a positive distance from $S$. Hence $(H(F, \theta, \phi)-E)^{-1}$ as well as $\left(H_{D}(F, \theta, \phi)-E\right)^{-1}$, where $H_{D}$ stands for an arbitrary cluster decomposition of $H$, are holomorphic and uniformly bounded over $|F| \geqq 0$ for $E \in S$. Now $S$ and the convergence regions of the rearrangements of the geometric expansions (4.1), (4.2) defining $I(F, \cdot), D(F, \cdot), I(\cdot), D(\cdot)$, have of course non empty intersection. Hence, by the known expression of the Weinberg kernels in terms of the resolvents of the cluster decompositions $H_{D}$ of $H,|F| \geqq 0$, by the above Lemma all assertions are a direct consequence of the analytic continuation principle and of the Vitali convergence theorem. This proves Lemma 4.5.

Proof of Theorem 4.1. By the same argument of Lemma 4.3 [the identity now being $\left.\left\{G(\theta) H(0,0, \phi) G(\theta)^{-1}-E\right\}^{-1} \equiv G(\theta)(H(0, \theta, \phi)-E)^{-1} G(\theta)^{-1}\right] E_{0}$ is an isolated eigenvalue, with the same (algebraic) multiplicity, also of $H(0, \theta, \phi)$, for $(\theta, \operatorname{Im} \phi)$ $\in \Theta_{\delta} x(-\alpha, \alpha)$. Let us now adapt Herbst's argument [5, Theorem III.3] to the present situation. Let $\eta<\arg (F)+\operatorname{Im} \phi<\pi-\eta$ for some $\eta>0$. Then there is $v>0$ such that the disk $C_{v}=\left\{E|| E-E_{0} \mid<v\right\}$ has the properties $\left\{E_{0}\right\}=C_{v} \cap \sigma(H(0, \theta, \phi))$, $d_{v}>0$, where $d_{v}$ is the distance between $C_{v}$ and the union over $(F, \theta, \phi)$ of the numerical ranges of $H_{0}(F, \theta, \phi)$. Consider first the three-body case $N=2$, and write the Weinberg-Van Winter equations for $(H(F, \theta, \phi)-E)^{-1},(H(0, \theta, \phi)-E)^{-1}$ :

$$
\begin{aligned}
& (H(F, \cdot)-E)^{-1}=D(F, \cdot, E)+I(F, \cdot, E)(H(F, \cdot)-E)^{-1}, \\
& (H(0, \cdot)-E)^{-1}=D(\cdot, E)+I(\cdot, E)(H(0, \cdot)-E)^{-1}
\end{aligned}
$$

valid for all $E \notin \sigma(H(F, \cdot)), E \notin \sigma(H(0, \cdot))$, respectively. Let us prove that $D(F, \cdot)$ and $I(F, \cdot)$ are holomorphic and bounded independently of $F$ for $E \in C_{v}$. For $N=2$ any cluster decomposition $H_{D}(F, \cdot)$ of $H(F, \cdot)$ with at least two clusters can always be written under the form

$$
H_{D}(F, \cdot)=h(F, \cdot) \otimes I+I \otimes h_{0}(F, \cdot) .
$$

Since $h(F, \cdot)$ and $h_{0}(F, \cdot)$ are both $m$-sectorial in $L^{2}\left(R^{3}\right)$, we can write (see Reed and Simon [11]):

$$
\left(H_{D}(F, \cdot)-E\right)^{-1}=(2 \pi i)^{-1} \int_{\Gamma}(h(F, \cdot)-\lambda)^{-1} \otimes\left(h_{0}(F, \cdot)-E+\lambda\right)^{-1} d \lambda .
$$

$\Gamma$ being the straight line $\operatorname{Im}(\lambda-E)=\operatorname{tg} \gamma(\operatorname{Re}(\lambda-E)-\varepsilon)$, where $\gamma=\arg (F)+\operatorname{Im} \phi$ $+\operatorname{Im} \theta / 2$, and $\varepsilon>0$ is such that $d\left(\Gamma, \sigma(h(0, \cdot))>v\right.$. By Lemma $4.3(h(F, \cdot)-\lambda)^{-1}$ is bounded uniformly with respect to $F$ for $\lambda \in \Gamma$ and $|F|$ small, and the same is true for $\left(h_{0}(F, \cdot)-E+\lambda\right)^{-1}$, uniformly for $E \in C_{v}$, because $-E+\lambda, E \in C_{v}, \lambda \in \Gamma$, has a positive distance from the union over $|F| \geqq 0$ of the numerical ranges of $h_{0}(F, \cdot)$. In addition by the uniform strict sectoriality of both $h(F, \cdot), h_{0}(F, \cdot)$ there are $M_{1}>0, M_{2}>0$ independent of $F$ such that $\left\|(h(F, \cdot)-\lambda)^{-1}\right\|<M_{1}|\lambda|^{-1}, \|\left(h_{0}(F, \cdot)\right.$ $-E+\lambda)^{-1} \|<M_{2}|\lambda|^{-1}$ as $|\lambda| \rightarrow \infty, \lambda \in \Gamma$. Hence the r.h.s. of (4.6) is uniformly norm convergent with respect to $F$ and $E \in C_{v}$, and thus the 1.h.s. is holomorphic for $E \in C_{v}$ and bounded independently of $F$ as required. Therefore $D(F, \cdot, E), I(F, \cdot, E)$ have the properties stated above, and by the Vitali theorem the convergence regions of $D(F, \cdot, E), I(F, \cdot, E)$ to $D(\cdot, E), I(\cdot, E)$, respectively, extend to $C_{v}$. In addition by $(4.4)(H(0, \cdot)-E)^{-1}=(I-I(\cdot, E))^{-1} D(\cdot, E)$ so that the above analyticity 
statement implies that $(I-I(\cdot, E))^{-1}$ is holomorphic for $E \varepsilon \partial C_{v}$. By the norm convergence $I(F, \cdot, E) \rightarrow I(\cdot, E)$, uniform on $C_{v}$, there is $F_{v}>0$ such that $(I-I(F, \cdot, E))^{-1}$ is holomorphic and uniformly bounded for $E \varepsilon \partial C_{v}, 0<|F|<F_{v}$. Since $D(F, \cdot, E)$ is holomorphic on $C_{v}$ and has the same uniform boundedness properties, $(H(F, \cdot)-E)^{-1}=(I-I(F, \cdot, E))^{-1} D(F, \cdot E)$ is holomorphic for $E \varepsilon \partial C_{v}$ and bounded uniformly over $F,|F|<F_{v}$. Hence (see Kato [9, VIII.1]) the strong convergence region of $(H(F, \cdot)-E)^{-1}$ to $(H(0, \cdot)-E)^{-1}$ as $|F| \rightarrow 0$ contains $\partial C_{v}$. Now set:

$$
P(F, \cdot)=(2 \pi i)^{-1} \int_{\partial C_{v}}(H(F, \cdot)-E)^{-1} d E, \quad P(0, \cdot)=(2 \pi i)^{-1} \int_{\partial C_{v}}(H(0, \cdot)-E)^{-1} d E
$$

By (4.3), (4.4) and the analyticity of $D(F, \cdot, E), D(\cdot, E)$ on $C_{v}$ we have:

$$
P(F, \cdot)-P(0, \cdot)=(2 \pi i)^{-1} \int_{\partial C_{v}}\left\{I(F, \cdot, E)(H(F, \cdot)-E)^{-1}-I(\cdot, E)(H(0, \cdot)-E)^{-1}\right\} d \mathrm{E} .
$$

The norm convergence of $I(F, \cdot, E)$ to $I(\cdot, E)$, uniform on $C_{v}$, is between compact operators so that the strong convergence, uniform on $\partial C_{v}$, of $(H(F, \cdot)-E)^{-1}$ to $(H(0, \cdot)-E)^{-1}$ yields, as $|F| \rightarrow 0,\left\|I(F, \cdot, E)(H(F, \cdot)-E)^{-1}-I(\cdot, E)(H(0, \cdot)-E)^{-1}\right\| \rightarrow 0$ with the stated uniformity in $(\theta, \phi)$ and uniformly for $E \varepsilon \partial C_{v}$. Substituting in (4.7) we get $\|P(F, \cdot)-P(0, \cdot)\| \rightarrow 0$ as $|F| \rightarrow 0$. Hence for $|F|$ suitably small (depending on $v) \operatorname{dim} P(F, \cdot)=\operatorname{dim} P(0, \cdot)$. Since $P(F, \cdot)$ characterizes the spectrum of $H(F, \theta, \phi)$ within $C_{v}$, and $v>0$ is arbitrary, the result is proved for $N=2$. The proof for all $N$ can now be obtained recursively. By the remark after Lemma 4.3, which holds in the $N=2$ case as well, the resolvent of any cluster decomposition $H_{D}$ of $H$, $N=3$, which can be expressed in terms of tensor products of $H, N=2, h, h_{0}$ if it has at least two clusters, can be uniformly controlled through (4.6) with $H, N=2$, in place of $h$ and $\varepsilon>-\inf (\sigma(h(0,0,0))-v)$. Hence $D(F, \cdot, E), I(F, \cdot, E)$ are holomorphic for $E \in C_{v}$ and bounded independently of $F$ for $N=3$. Then we can repeat the above argument for $N=3$, and iterate it to conclude the proof for all $N$.

Corollary 4.6. Let $\operatorname{dim} P(0, \cdot)=1$, and let $E_{0}(F)$ be the eigenvalue of $H(F, \cdot)$ close to $E_{0}$. Then there are $B>0, \eta>0$ such that $E_{0}(F)$ is analytic in the region

$$
C_{0}=\{F|0<| F|<B|-\pi / 2+\eta<\arg (F)<3 \pi / 2-\eta\} .
$$

Theorem 4.7. Let $E_{0}(F)$ be as in Corollary 4.6, and $E_{0}$ be the corresponding simple eigenvalue of $H(0,0,0)$. Then the Rayleigh-Schrödinger perturbation expansion $\sum_{0}^{\infty} c_{k} F^{k}\left(c_{0}=E_{0}\right)$ near $E_{0}$ exists and is strongly asymptotic to $E_{0}(F)$ for all $F$ in $\{F|0<| F|<B|-\pi / 2+\varepsilon<\arg (F)<3 \pi / 2-\varepsilon\}, \varepsilon>0$, i.e. there are $A>0, A^{\prime}>0$ such that, uniformly with respect to $F$ as above:

$$
\left|E_{0}(F)-\sum_{0}^{N} c_{k} F^{k}\right|<A^{\prime} A^{N+1}(N+1) !|F|^{N+1} .
$$

Proof. For $B$ small enough we can write $E_{0}(F)=N(F) / D(F)$, where

$$
\begin{gathered}
N(F)=(2 \pi i)^{-1} \int_{\partial C_{v}} E<\psi_{0}(\bar{\theta}, \bar{\phi}), G(\theta)[H(F, \theta, \phi)-E]^{-1} G(\theta)^{-1} \psi_{0}(\theta, \phi)>d E \\
D(F)=(2 \pi i)^{-1} \int_{\partial C_{v}}<\psi_{0}(\bar{\theta}, \bar{\phi}), G(\theta)[H(F, \theta, \phi)-E]^{-1} G(\theta)^{-1} \psi_{0}(\theta, \phi)>d E .
\end{gathered}
$$


$\psi_{0}(\theta, \phi)$ being the eigenvector of $H(0, \theta, \phi)$ corresponding to $E_{0}$. Now, $\psi_{0}(\phi)$ being the eigenvector of $H(F, 0, \phi)$ corresponding to $E_{0}$, by Theorem $3.6(\mathrm{v})$ one has

$$
\begin{aligned}
& \left\langle\psi_{0}(\bar{\theta}, \bar{\phi}), G(\theta)[H(F, \theta, \phi)-E]^{-1} G(\theta)^{-1} \psi_{0}(\theta, \phi)\right\rangle \\
& \quad=\left\langle\psi_{0}(\bar{\phi}),[H(F, 0, \phi)-E]^{-1} \psi_{0}(\phi)\right\rangle .
\end{aligned}
$$

The second Neumann expansion of $[H(F, 0, \phi)-E]^{-1}$ yields, formally:

$$
\begin{aligned}
{[H(F, 0, \phi)-E]^{-1}=} & {[H(0,0, \phi)-E]^{-1}\left\{\sum_{0}^{N}\left(e^{\phi} F z_{N}(H(0,0, \phi)-E)^{-1}\right)^{n}\right.} \\
& \left.+[H(F, 0, \phi)-E]^{-1}\left[e^{\phi} F z_{N}(H(0,0, \phi)-E)^{-1}\right]^{N+1}\right\}, \\
& N=1,2, \ldots
\end{aligned}
$$

Let us now prove the bound $\left\|\left(z_{N}[H(0,0, \phi)-E]^{-1}\right)^{n} \psi_{0}(\phi)\right\|<A_{1}^{\prime} A_{1}^{n} n$ !, for some $A_{1}^{\prime}>0, A_{1}>0$, uniformly with respect to $E \in \partial C_{v}$, by means of a suitable modification of Herbst's argument (Theorem III.3 of [5]). First remark that by the CombesThomas theory (see e.g. [10, XIII.11]) $\psi_{0}(\phi) \in D\left(e^{\gamma r}\right)$ for some $\gamma>0, r=|x|$. It is easily shown that the operators

$$
R_{E} \equiv[H(0,0, \phi)-E]^{-1}, \quad B(\beta, E) \equiv\left[e^{\beta r} z_{N}, R_{E}\right] e^{-\beta r}\left(i \gamma^{-1}+z_{N}\right)^{-1}, \quad 0<\beta+\gamma,
$$

are uniformly bounded for $E \in \partial C_{v}, \phi$ as above. Therefore there are $d_{1}>0, d_{2}>0$ such that $\left\|R_{E}\right\|<d_{1},\|B(\beta, E)\|<d_{2}$. Note also the obvious inequality $\left\|z_{N} e^{-\beta r}\right\|$ $<(\beta e)^{-1}$. Let now $\psi_{n-k}=e^{n^{-1} k \gamma r}\left(z_{N} R_{E}\right)^{n-k} \psi_{0}, k=0, \ldots, n-1$. The identity

$$
\psi_{n-k}=R_{E^{2}} e^{-n^{-1} \gamma r} \psi_{n-k-1}+B\left(n^{-1} k \gamma, E\right)\left(i \gamma^{-1}=z_{N}\right) e^{-n^{-1} \gamma r} \psi_{n-k-1}
$$

yields $\left\|\psi_{n-k}\right\|<\left(d_{1}(e \gamma)^{-1} n+d_{2}(e \gamma)^{-1}(n+1)\right)\left\|\psi_{n-k-1}\right\|$, and since $\psi_{n}=\left(z_{N} R_{E}\right)^{n} \psi_{0}$, we have $\left\|\left(z_{N} R_{E}\right)^{n} \psi_{0}\right\| \leqq(e \gamma)^{-n}\left(d_{1}+d_{2}\right)^{n}(n+1)^{n}\left\|e^{\gamma r} \psi_{0}\right\| \leqq A_{1}^{\prime} A_{1}^{n} n$ ! for some $A_{1}^{\prime}>0$, $A_{1}=(e \gamma)^{-1}\left(d_{1}+d_{2}\right)$. Since $[H(F, 0, \phi)-E]^{-1}$ is uniformly bounded for $E \in \partial C_{v}$ in the stated regions of $F$ and $\phi$, we have

$$
\begin{aligned}
& \left\|\{H(0,0, \phi)-E\}^{-1}\left\{z_{N} e^{\phi}[H(0,0, \phi)-E]^{-1}\right\}^{n} \psi_{0}(\phi)\right\| \leqq B_{1} A_{1}^{n} n ! \\
& \left\|\{H(F, 0, \phi)-E\}^{-1}\left\{z_{N} e^{\phi}[H(0,0, \phi)-E]^{-1}\right\}^{N+1} \psi_{0}(\phi)\right\| \leqq B_{2} A_{1}^{N+1}(N+1) !
\end{aligned}
$$

for some positive $B_{1}$ and $B_{2}$ independent of $E$. Hence $N(F)$ as well as $D(F)$ satisfy a strong asymptotic condition of the type (4.2). Since $\psi_{0}(F, \phi) \neq 0$ for $|F|$ small enough, it follows (see e.g. [10, XII.6]) the existence of $A^{\prime}, A>0$ such that $E_{0}(F)$ $=N(F) / D(F)$ satisfies the strong asymptotic condition (4.2). This proves Theorem 4.6.

Corollary 4.8. Let $E_{0}(F)$ be as above. Then the Rayleigh-Schrödinger perturbation expansion near $E_{0}$ is Borel summable to $E_{0}(F)$ in the sector $\left\{F|0<| F\left|<B_{0}\right| \eta<\arg (F)\right.$ $<\pi-\eta\}$, for some $B_{0}>0, \eta>0$.

Proof. A direct consequence of the Watson theorem (see e.g. [10, XII.6]) whose conditions are verified by Corollary 4.6 and Theorem 4.7.

Remarks. (i) The Borel transform $B(F)=\sum_{0}^{\infty} c_{k} F^{k} / k$ ! of $E_{0}(F)$ is analytic for $|F|<B_{0}$ and has an analytic continuation to the sector $|\arg (F / i)|<\eta$ such that $\int_{0} e^{-t}|B(z t)| d t$ 
$<\infty$ for $|F|<B_{0},|\arg (F / i)|<\eta$. Hence the Borel sum of the series, given by definition by the above integral, coincides with $E_{0}(F)$ for $0<|F|<B_{0},|\arg (F / i)|<\eta$. The analytic continuation of $E_{0}(F)$ for $|F|$ small can be performed directly on the expression of the Borel sum (see Sokal [13]), which can be written in the form: $E_{0}(F)=e^{i \alpha} \int_{0}^{\infty} e^{-t e^{i \alpha}} B\left(F t e^{i \alpha}\right) d t$ for $|F|<B_{0} \cos \alpha, \eta<\alpha<\pi / 2$.

The last integral converges absolutely for $\left|\arg \left(e^{i \alpha} F / i\right)\right|<\eta$, and hence defines the analytic continuation of $E_{0}(F)$ for all $F$ in $0<|F|<B_{0} \pi / 2-\alpha-\eta<\arg (F)$ $<\pi / 2+\eta-\alpha$. The real axis $\arg (F)=0$ is included if we take $0<\alpha<\pi / 2-\eta$.

(ii) If, as in Theorem 9 of [8] $\operatorname{Im} E_{0}(F), F$ real, is strictly negative, we can define the resonance width $\Gamma(F)=-2 \operatorname{Im} E_{0}(F)>0$, and the perturbation theory diverges. If in addition, again as in Theorem 9 of [9], $V\left(-z_{1}, \ldots,-z_{N}\right)=V\left(z_{1}, \ldots, z_{N}\right)$, since the remaining assumptions of that result are verified by Corollaries 4.6 and 4.8 , we have: $c_{2 k+1}=0$, all $k ; c_{2 k}=-\pi^{-1} \int_{0}^{R} \Gamma(x) x^{-(2 k+1)} d x+O\left(R^{-2 k}\right)$, for $k \rightarrow \infty$ and some $R>O$. In particular, $\operatorname{Im} E_{0}(F)$ is $o\left(F^{k}\right)$ for all $k$ as $F \rightarrow 0$.

\section{References}

1. Aguilar, J., Combes, J.M.: Commun. Math. Phys. 22, 269 (1971)

2. Balslev, E., Combes, J.M.: Commun. Math. Phys. 22, 280 (1971)

3. Graffi, S., Grecchi, V.: Commun. Math. Phys. 62, 83 (1978)

4. Hardy, G.H.: Divergent Series. Oxford:Oxford University Press 1947

5. Herbst, I.W.: Commun. Math. Phys. 64, 279 (1979)

6. Herbst, I.W., Simon, B.: Phys. Rev. Lett. 41, 67 (1978)

7. Herbst, I.W., Simon, B.: Dilation analyticity in constant electric field. II. The $n$-body case, borel summability (in preparation)

8. Hunziker, W.: Schrödinger operators with magnetic and electric fields. Proceedings of the international conference of the association of the mathematical physicists. Lausanne: Springer 1979

9. Kato, T. : Perturbation theory of linear operators. Berlin, Heidelberg, New York: Springer 1966 (2nd ed. 1976)

10. Reed, M., Simon, B.: Methods of modern mathematical physics, Vols. 1, 2, 4. New York, London: Academic Press 1975, 1976, 1978

11. Reed, M., Simon, B. : J. Funct. Anal. 13, 107 (1973)

12. Simon, B.: Ann. Phys. 58, 76 (1970)

13. Sokal, A.: An improvement of watson's theorem on borel summability. Princeton: Princeton University Preprint 1979

Communicated by J. Ginibre

Received March 10, 1980 
